\title{
Extreme submillimetre starburst galaxies
}

\author{
M. Rowan-Robinson ${ }^{1}$, Lingyu Wang ${ }^{2,3}$, Duncan Farrah ${ }^{4,5}$, Dimitra Rigopoulou ${ }^{6}$, Carlotta Gruppioni ${ }^{7}$, \\ Mattia Vaccari ${ }^{8,10}$, Lucia Marchetti ${ }^{8,9}$, David L. Clements ${ }^{1}$, and William J. Pearson ${ }^{2,3}$ \\ 1 Astrophysics Group, Imperial College London, Blackett Laboratory, Prince Consort Road, London SW7 2AZ, UK \\ e-mail: mrr@imperial.ac.uk \\ 2 SRON, Groningen 9747, AD, the Netherlands \\ 3 Kapteyn Astronomical Institute, University of Groningen, Groningen 9747, AD, the Netherlands \\ 4 Department of Physics and Astronomy, University of Hawaii, 2505 Correa Road, Honolulu, HI 96822, USA \\ 5 Institute for Astronomy, University of Hawaii, 2680 Woodlawn Drive, Honolulu, HI 96822, USA \\ ${ }^{6}$ Department of Astrophysics, University of Oxford, Keble Rd, Oxford OX1 3RH, UK \\ 7 INAF, Osservatorio Astronomico di Bologna, Via Ranzani 1, 40127 Bologna, Italy \\ 8 Department of Physics and Astronomy, University of the Western Cape, Robert Sobukwe Road, 7535 Bellville, \\ Cape Town, South Africa \\ 9 Department of Astronomy, University of Cape Town, Rondebosch 7701, South Africa \\ 10 INAF, Istituto di Radioastronomia, Via Gobetti 101, 40129 Bologna, Italy
}

Received 19 January 2018 / Accepted 9 September 2018

\begin{abstract}
We have used two catalogues, a Herschel catalogue selected at $500 \mu \mathrm{m}$ (HerMES) and an IRAS catalogue selected at $60 \mu \mathrm{m}$ (RIFSCz), to contrast the sky at these two wavelengths. Both surveys demonstrate the existence of "extreme" starbursts, with star-formation rates (SFRs) $>5000 M_{\odot} \mathrm{yr}^{-1}$. The maximum intrinsic star-formation rate appears to be $\sim 30000 M_{\odot} \mathrm{yr}^{-1}$. The sources with apparent SFR estimates higher than this are in all cases either lensed systems, blazars, or erroneous photometric redshifts. At redshifts between three and five, the time-scale for the Herschel galaxies to make their current mass of stars at their present rate of star formation is $\sim 10^{8} \mathrm{yr}$, so these galaxies are making a significant fraction of their stars in the current star-formation episode. Using dust mass as a proxy for gas mass, the Herschel galaxies at redshift three to five have gas masses comparable to their mass in stars. Of the 38 extreme starbursts in our Herschel survey for which we have more complete spectral energy distribution (SED) information, 50\% show evidence for QSO-like optical emission, or exhibit AGN dust tori in the mid-infrared SEDs. In all cases however the infrared luminosity is dominated by a starburst component. We derive a mean covering factor for AGN dust as a function of redshift and derive black hole masses and black hole accretion rates. There is a universal ratio of black-hole mass to stellar mass in these high redshift systems of $\sim 10^{-3}$, driven by the strong period of star-formation and black-hole growth at $z=1-5$.
\end{abstract}

Key words. galaxies: evolution - infrared: galaxies - galaxies: star formation - quasars: supermassive black holes galaxies: starburst - cosmology: observations

\section{Introduction}

A key discovery from the Infrared Astronomical Satellite (IRAS) surveys was the existence of galaxies with remarkably high infrared (rest-frame $1-1000 \mu \mathrm{m}$ ) luminosities. IRAS found thousands of galaxies with infrared luminosities $>10^{12} L_{\odot}$, termed "ultraluminous" infrared galaxies or ULIRGs (Soifer et al. 1984; Aaronson \& Olszewski 1984; Houck et al. 1985; Joseph \& Wright 1985; Allen et al. 1985; Lawrence et al. 1986), and over a hundred with luminosities $>10^{13} L_{\odot}$, termed "hyperluminous" infrared galaxies or HyLIRGs (Rowan-Robinson 2000; Rowan-Robinson $\&$ Wang 2010). All-sky surveys in the mid-infrared with WISE also uncovered comparably luminous systems (Eisenhardt et al. 2012). While rare locally, infrared-luminous systems rise dramatically in number with increasing redshift, until at $z>1$ they host a substantial, possibly dominant fraction of the comoving infrared luminosity density (Le Floc'h et al. 2005; Perez-Gonzalez et al. 2005). Infrared template modelling, and other follow-up, has shown that both starbursts and AGN dust tori can contribute to these very high infrared luminosities, with implied star formation rates (SFRs) exceeding $1000 M_{\odot} \mathrm{yr}^{-1}$. Selection at 22 (WISE) or 25 (IRAS) $\mu \mathrm{m}$ favours dominance by AGN dust tori, while selection at $60 \mu \mathrm{m}$ or longer wavelengths favours dominance by star formation. In order to obtain the most robust selection possible we here use infrared template modelling to select sources on the basis of their star-formation rate, rather than their infrared luminosity.

More recent surveys at longer wavelengths by Herschel have drawn attention to even more extreme objects, with starformation rates in excess of $10000 M_{\odot} \mathrm{yr}^{-1}$ in some cases (Rowan-Robinson et al. 2016). The existence of these "extreme" starbursts poses a fundamental problem for semi-analytic models of galaxy formation. The observed number density of extreme starbursts with SFRs $>1000 M_{\odot} \mathrm{yr}^{-1}$ (Dowell et al. 2014; Asboth et al. 2016) is factors of several above model predictions, while the extreme starbursts with SFRs $>3000 M_{\odot} \mathrm{yr}^{-1}$ do not exist at all in models (Lacey et al. 2010, 2016; Gruppioni et al. 2011, 2015; Hayward 2013; Henriques et al. 2015). The issue for the models is that neither mergers or cold accretion should produce such high SFRs; mergers because they cannot channel enough gas to the centers of haloes (e.g. Fig. 1 of Narayanan et al. 2010; Dave et al. 2010), and cold accretion because massive haloes inhibit the gas flow on to central galaxies via shock heating 
(Birnboim \& Dekel 2003; Keres et al. 2005; Narayanan et al. 2015). The models could potentially reproduce SFRs of $>3000 M_{\odot} \mathrm{yr}^{-1}$ at $z>1$ if feedback is turned off completely, but would then strongly overpredict the $z=0$ galaxy mass function.

There is thus a pressing need to confirm the existence of systems with such high star formation rates, especially at high redshifts, understand how efficient surveys at different wavelengths are at uncovering them, and to understand the relation between their stellar mass and black hole mass assembly events. In this paper we undertake such a study, by examining and contrasting the selection of extreme starburst galaxies from two surveys, one at $60 \mu \mathrm{m}$ and one at $500 \mu \mathrm{m}$. There are four reasons for using a $60 \mu \mathrm{m}$ (IRAS) sample as well as a $500 \mu \mathrm{m}$ one. Firstly the contrast between 500 and $60 \mu \mathrm{m}$ surveys brings out what is distinct about the $500 \mu \mathrm{m}$ sky. Secondly we find that the $60 \mu \mathrm{m}$ sample helps us delineate the maximum possible rate of star-formation in galaxies (Sect. 4). Thirdly our $60 \mu \mathrm{m}$ survey is free of the problems of confusion and blending which are issues at submillimetre wavelengths, because of the small numbers of sources per beam. Confusion only became an issue for IRAS in the Galactic plane and in the very deepest IRAS surveys at the north ecliptic pole (Hacking \& Houck 1987). Finally in our analysis of AGN (Sect. 7) the $60 \mu \mathrm{m}$ sample provides us with a useful low redshift benchmark.

This paper is structured as follows. Section 2 describes our sample selection strategy from the IRAS and Herschel surveys. In Sect. 4 we outline how candidate lenses are removed from the samples. We then describe how stellar masses, gas masses, and star formation rates are computed for each source in Sect. 5. Using these derived quantities, we then examine the properties of the extreme starbursts in the sample in Sect. 6, and the role of AGN in Sect. 7.

A cosmological model with $\Lambda=0.7, h_{0}=0.72$ has been used throughout. If we were to use $H_{0}=67 \mathrm{~km} \mathrm{~s}^{-1} \mathrm{Mpc}$ (Planck Collaboration XVI 2014) then luminosities and star-formation rates would increase by $15.5 \%$.

\section{Sample Selection}

We have selected sources from two catalogs; the Revised IRAS Faint Source Survey Redshift Catalogue (RIFSCz; Wang et al. 2014a), and the Herschel Multi-tiered Extragalactic Survey (HerMES; Oliver et al. 2012). Below, we describe each catalogue in turn.

\subsection{IRAS}

The Revised IRAS Faint Source Survey Redshift (RIFSCz) Catalogue (Wang et al. 2014a) is a $60 \mu \mathrm{m}$ survey for galaxies over the whole sky at $|b|>20^{\circ}$, which incorporates data from the SDSS, 2MASS, WISE, and Planck all-sky surveys to give wavelength coverage from 0.36 to $1380 \mu \mathrm{m}$. Since publication of Wang et al. (2014a) AKARI fluxes have been added to the catalogue, using a search radius of 1 arc min. An aperture correction needs to be applied to AKARI 65 and $90 \mu$ m fluxes to give consistency with IRAS photometry (Rowan-Robinson \& Wang 2017). Furthermore the optical and near-infrared photometry of 1271 catalogued nearby galaxies has been improved, following a systematic trawl through the NASA/IPAC Extragalactic Database. Wang et al. (2014a) found that $93 \%$ of RIFSCz sources had optical or near infrared counterparts with spectroscopic or photometric redshifts. The photometric redshifts primarily make use of 2MASS and SDSS photometric data. Thus for $93 \%$ of the catalogue the prime selection effect
Table 1. RIFSCz catalogue by band.

\begin{tabular}{lll}
\hline \hline $\begin{array}{l}\text { Wavelength } \\
(\mu \mathrm{m})\end{array}$ & Survey & $\begin{array}{l}\text { Number of } \\
\text { sources }\end{array}$ \\
\hline 3.4 & WISE & 48603 \\
4.6 & WISE & 48603 \\
12 & WISE & 48591 \\
12 & IRAS & 4476 \\
22 & WISE & 48588 \\
25 & IRAS & 9608 \\
60 & IRAS & 60303 \\
65 & AKARI & 857 \\
90 & AKARI & 18153 \\
100 & IRAS & 30942 \\
140 & AKARI & 3601 \\
160 & AKARI & 739 \\
350 & Planck & 2275 \\
550 & Planck & 1152 \\
850 & Planck & 616 \\
1380 & Planck & 150 \\
\hline
\end{tabular}

is the $60 \mu \mathrm{m}$ sensitivity limit of the IRAS Faint Source Survey $(\sim 0.36 \mathrm{mJy})$. Table 1 summarises the number of RIFSCz galaxies by waveband.

\subsection{Herschel}

The HerMES survey allows us to construct a $500 \mu \mathrm{m}$ sample of galaxies in areas in which we have deep optical and infrared data from the Spitzer-SWIRE survey (Lonsdale et al. 2003; RowanRobinson et al. 2008, 2014, 2016) over a total area of $26.3 \mathrm{sq}$ deg in five fields (see Table 1 of Rowan-Robinson et al. 2016). Aperture corrections are applied at optical, near and mid-infrared wavelengths to ensure that all SEDs are based on integrated fluxdensities (Rowan-Robinson et al. 2013). Selection at $500 \mu \mathrm{m}$, rather than say $250 \mu \mathrm{m}$, gives us greater visibility of the high redshift $(z>3)$ universe due to the intrinsic shape of starburst SEDs at far-infrared wavelengths (Franceschini et al. 1991) and has the benefit of ensuring detection also at 350, and in most cases $250 \mu \mathrm{m}$, to give valuable SED information. The association of Herschel sources with SWIRE $24 \mu \mathrm{m}$ sources uses a likelihood which combines the positional disagreement between $250 / 350 \mu \mathrm{m}$ and $24 \mu \mathrm{m}$ positions and the agreement of the observed $500 \mu \mathrm{m}$ flux with that predicted from automatic template fits to the SWIRE $4.5-170 \mu \mathrm{m}$ data. We have argued previously (Rowan-Robinson et al. 2014) that the use of SED information is essential in the association process. Assignment of submillimetre flux to counterparts based purely on positional agreement can lead to physically unrealistic SEDs. The complete HerMESSWIRE $500 \mu \mathrm{m}$ catalogue comprises sources in the Lockman, XMM, ELAIS-S1, ELAIS-N1 and CDF-S fields, and consists of 2181 galaxies. In the Lockman+XMM+ELAIS-S1 areas there are a further 833 good quality $500+350 \mu \mathrm{m}$ sources which are not associated with Spitzer-SWIRE galaxies, for which RowanRobinson et al. (2016) have estimated redshifts from their submillimetre colours. Thus for all HerMES $500 \mu \mathrm{m}$ sources we have an estimate of redshift and hence of infrared luminosity (and star-formation rate). The prime selection effect on this sample is therefore the $500 \mu \mathrm{m}$ flux-density limit of the survey.

We performed a check of the surface density of $500 \mu \mathrm{m}$ sources in the HerMES survey using data from the COSMOS area (Scoville et al. 2007), which was surveyed as part of the 
Table 2. Contrast between the $60 \mu \mathrm{m}$ selected RIFSCz catalogue and the $500 \mu \mathrm{m}$ selected catalogue from the HerMES survey.

\begin{tabular}{lll}
\hline \hline & IRAS-FSS & HerMES-SWIRE \\
& $60 \mu \mathrm{m}$ & $500 \mu \mathrm{m}$ \\
\hline Number of sources & 60303 & 2181 \\
Effective area (sq deg) & 27143 & 26.3 \\
Surface-density of lensed galaxies & $0.001 \mathrm{per} \mathrm{deg}^{2}$ & $10 \mathrm{per} \mathrm{deg}^{2}$ \\
Fraction of Ultraluminous galaxies & $8 \%$ & $70 \%$ \\
Fraction of Hyperluminous galaxies & $0.7 \%$ & $25 \%$ \\
Fraction of galaxies with standard cirrus & $42 \%$ & $34 \%$ \\
Fraction of galaxies with cool or cold cirrus & $2.5 \%$ & $29 \%$ \\
Redshift $>0.3$ & $4 \%$ & $88 \%$ \\
\hline
\end{tabular}

HerMES project. Photometric redshifts for COSMOS have been discussed by Ilbert et al. (2013) and Laigle et al. (2016). There are $181500 \mu \mathrm{m}$ sources with flux greater than $25 \mathrm{mJy}$, the flux limit we used in Rowan-Robinson et al. (2016), and which also have $350 \mu \mathrm{m}$ detections, in the $2.0 \mathrm{sq}$ deg of the COSMOS survey. All have $24 \mu \mathrm{m}$ associations. This yields a $500 \mu \mathrm{m}$ sourcedensity of 90 per sq deg, similar to that found in the $26.3 \mathrm{sq} \mathrm{deg}$ of our sample.

\subsection{Comparison with other studies}

Schulz et al. (2017) have published a new IPAC SPIRE catalogue (HPSC) which analyses data taken in all Herschel-SPIRE programmes in a homogeneous way, using a blind source detection approach. This would appear to offer the opportunity of a much larger sample of SPIRE galaxies. We used the HPSC catalogue to create a 250-350-500 $\mu \mathrm{m}$ list as in Rowan-Robinson et al. (2014). When we associated this list with the SWIRE photometric redshift catalogue (Rowan-Robinson et al. 2013), we found only about half of the 2181 sources. This is an issue acknowledged in the HPSC explanatory supplement, which they attribute to blending of SPIRE sources in their detection procedure.

We also associated this HPSC $500 \mu \mathrm{m}$ catalogue with RIF$\mathrm{SCz}$, finding 1640 associations. Many of these were also detected by Planck and so we can make a direct comparison of 350 and $500 \mu \mathrm{m}$ fluxes in the two surveys. The sources in common to HPSC, RIFSCz and Planck tend to be low redshift galaxies. We find that these galaxies need an aperture correction of $k^{*}$ delmag to the SWIRE fluxes, where delmag $=J_{\text {ext }}-J_{\mathrm{ps}}$ is the $J$-band aperture correction and $k=0.15$ at 350 , and 0.10 at $500 \mu \mathrm{m}$, to get agreement of SPIRE and Planck fluxes. Previously Wang et al. (2014a) reported the need for aperture corrections to be applied to WISE fluxes at 12 and $22 \mu \mathrm{m}$. The latest version of RIFSC $z^{1}$ thus provides a comprehensive collection of fluxes, with aperture corrections where necessary, from optical (SDSS), near infrared (2MASS), mid and far infrared (WISE, IRAS, AKARI), through to submillimetre and millimetre (Herschel and Planck).

Koprowski et al. (2017) have used a SCUBA2 survey at $850 \mu \mathrm{m}$ to estimate the rest-frame $250 \mu \mathrm{m}$ luminosity-density and then translated this to a star-formation-rate-density assuming a universal submillimetre SED. They cast doubt on the reality of the high star-formation rates found by Rowan-Robinson et al. (2016) at redshift four to six. There are some flaws in the Koprowski et al analysis. Firstly their $850 \mu \mathrm{m}$ detection threshold is set at $3.5 \sigma$, which means they are heavily into the confu-

\footnotetext{
1 http://mattiavaccari.net:/df/mrr/readmeRIFSCz
}

sion regime. Our strategy of thresholding at $5 \sigma$, made possible by the excellent submillimetre sensitivity of Herschel-SPIRE, ensures that problems of confusion and source blending are greatly reduced (see Sect. 3). Secondly, they associate their submillimetre sources with other multi-wavelength data using the nearest bright 8 or $24 \mu \mathrm{m}$, or $1.4 \mathrm{GHz}$, source, thus potentially biassing their associations against more probable (in terms of their SED) higher redshift galaxies. Finally because their survey is at a single submillimetre wavelength they have no reliable way of estimating the star-formation rate. It is simply not true that all submillimetre galaxies have a common submillimetre SED (e.g. Rowan-Robinson et al. 2014). The high star-formation rates we find are supported by the IRAS RIFSCz sample (see Sect. 5 below) which is not subject to any of the submillimetre confusion or blending issues. Novak et al. (2017) have nicely confirmed Rowan-Robinson et al. (2016)'s star-formation-ratedensity from redshift zero to five with radio estimates from a VLA survey.

Table 2 shows a comparison of the sky seen at 60 and at $500 \mu \mathrm{m}$, as seen in the RIFSCz and HerMES-SWIRE catalogues (Rowan-Robinson et al. 2014). The most striking contrasts of $500 \mu \mathrm{m}$ selection, compared to $60 \mu \mathrm{m}$ selection, are (i) a much higher fraction of high redshift galaxies (as predicted by Franceschini et al. 1991), (ii) a much higher fraction of lensed objects (as predicted by Blain et al. 2002), (iii) a much higher fraction of galaxies with cool or cold dust (Rowan-Robinson \& Wang 2010; Rowan-Robinson et al. 2016; Rowan-Robinson \& Clements 2015).

\section{Confusion and source blending}

An important issues for ground- and space-based submillimetre surveys is confusion and source-blending. For a random distribution of point-sources characterised by differential source-counts $\mathrm{d} N / \mathrm{d} S=n(S)$, where $S$ is the flux-density, observed with a telescope of specified beam, the measured responses are characterised by the probability of an observed deflection $D, P(D)$. Scheuer (1957) gave the formalism for calculating $P(D)$ and Condon (1974) used this to calculate the confusion noise for a telescope with a Gaussian beam of dispersion $\Theta$ and a powerlaw source-count distribution $n(S)=k S^{-\gamma}$. Integrating $S^{2} P(D)$ from $S=0$ to $D_{\text {c }}$ to evaluate the rms dispersion $\sigma^{2}$ gives

$\sigma=\left(k \Omega_{\mathrm{e}} /(3-\gamma)^{1 / 2} D_{\mathrm{c}}^{(3-\gamma) / 2}\right.$

where the effective telescope beam $\Omega_{\mathrm{e}}=\Omega /(\gamma-1)$ and the Gaussian beam area

$\Omega=2 \pi \Theta^{2}=1.14 \Theta_{\mathrm{FWHP}}^{2}$ 
Table 3. Number of beams per source for 5- $\sigma$ survey, as function of $\gamma$.

\begin{tabular}{llllll}
\hline \hline$\gamma=$ & 1.5 & 2.5 & 2.6 & 2.7 & 2.8 \\
No. of beams per source $=$ & 17 & 50 & 61 & 83 & 125 \\
\hline
\end{tabular}

Thresholding at a multiple $q$ of $\sigma, D_{\mathrm{c}}=q \sigma$, yields

$\sigma=\left(q^{(3-\gamma)} /(3-\gamma)\right)^{1 /(\gamma-1)}\left(k \Omega_{\mathrm{e}}\right)^{1 /(\gamma-1)}$

(Condon's Eq. 14). We can use this to calculate the number of sources per Gaussian beam at the $q \sigma$ limit (after some cancellation):

$N(q \sigma) \Omega=(3-\gamma) / q^{2}$.

Results for $q=5$ and different values of $\gamma$ are given in Table 3 . Franceschini (1982) has an expression equivalent to Eq. (4) in his Eq. (14).

Hacking \& Houck (1987) repeated the Condon calculation and gave a table of results on $\alpha=\gamma-1$, the beam size and $\sigma$ for their deep 12 and $60 \mu \mathrm{m}$ survey. They confirm that at $60 \mu \mathrm{m}$, where $\gamma=2.5$, their survey is confusion limited at their 5- $\sigma$ limit of $50 \mathrm{mJy}$, at a source-density of 1 source per 50 beams. Hogg (2001) carried out simulations of position error, flux error and completeness for $\alpha=1-1.5$ (relevant to optical galaxy counts. He gives a rule of thumb of 30 beams per source for avoiding effects of confusion, but finds that for $\alpha \geq 1.5$, the requirement should be 50 beams per source.

For applications to Herschel $500 \mu \mathrm{m}$ surveys we note that $\Theta_{\mathrm{FWHM}}=36.6 \mathrm{arcsec}$, so $\Omega=1 / 8486 \mathrm{sq} \mathrm{deg}$. From Bethermin et al. (2012)'s $500 \mu \mathrm{m}$ differential source-counts we find the source count slope at 6-24 mJy $(\sim 1-5 \sigma)$ is $\gamma \sim 2.65$, so Eq. (1) predicts the 5- $\sigma$ limit as one source per 71 beams. In Rowan-Robinson et al. (2014) we found 1335 5- $\sigma 500 \mu \mathrm{m}$ sources in the $7.53 \mathrm{sq}$ deg of the Lockman-SWIRE area, which would correspond to one source per 48 beams. Of course the true source-counts can not be a power-law at all fluxes and this would modify the calculation slightly.

We also calculate the probability of a blend of a source fS with a second source $(1-f) S$, where $S=5 \sigma$, within the telescope beam, for an assumed source-count slope $\gamma=2.65$ and find

$p(f S,(1-f) S)=\left[(3-\gamma) / q^{2}\right]^{2} f^{-\gamma+1}(1-f)^{-\gamma+1}$.

Values of the relative probability of different blending cases, $p(f S,(1-f) S) / p(S)$, for different values of $\mathrm{f}$ are given in Table 4 for 500 and $350 \mu \mathrm{m}$. A similar expression can be derived for blends of three sources, $p(f S, g S,(1-f-g) S)$, and values for the relative probability of three-source blends are also given in Table 4.

The probability of a roughly equal-flux blend is extremely low at $500 \mu \mathrm{m}$ and even lower at $350 \mu \mathrm{m}$, where all the sources have to be detected to be in our sample. Most of the sources are also detected at $250 \mu \mathrm{m}$, where the probabilities are lower still, by a further factor of two.

These probabilities apply to an unclustered distribution of sources. Source confusion will be enhanced by intrinsic clustering of galaxies (Barcons 1992; Scott et al. 2002; Bethermin et al. 2017).

Scott et al. (2002), carried out simulations of the SCUBA $850 \mu \mathrm{m} 8 \mathrm{mJy}$ survey. From their tables we see that to achieve better than $90 \%$ completeness, positional error $<20 \%$ of the beam width, and flux-boost $<5 \%$, we need to threshold at
Table 4. Relative probability of two-source blends, $p(f S,(1-$ $f) S) / p(S)$, and three-source blends, $p(f S, g S,(1-f-g) S) / p(S)$, where $S=5 \sigma$ and $\gamma=2.65$.

\begin{tabular}{llll}
\hline \hline & $500 \mu \mathrm{m}$ & $350 \mu \mathrm{m}$ & $250 \mu \mathrm{m}$ \\
\hline Two-source blends & & & \\
$0.8 S, 0.2 S$ & 0.29 & 0.145 & 0.07 \\
$0.7 S, 0.3 S$ & 0.18 & 0.09 & 0.045 \\
$0.6 S, 0.4 S$ & 0.15 & 0.075 & 0.04 \\
$0.5 S, 0.5 S$ & 0.14 & 0.07 & 0.035 \\
Three-source blends & & & \\
$0.6 S, 0.2 S, 0.2 S$ & 0.09 & 0.045 & 0.02 \\
$0.4 S, 0.3 S, 0.3 S$ & 0.05 & 0.025 & 0.012 \\
\hline
\end{tabular}

5- $\sigma$. Michalowski et al. (2017) found through ALMA followup that the fraction of bright SCUBA $850 \mu \mathrm{m}$ sources $\left(S_{850}>\right.$ $4 \mathrm{mJy}$ ) significantly affected by blending is small (15-20\%). Hill et al. (2018) have observed 103 bright SCUBA $850 \mu \mathrm{m}$ sources $\left(S_{850}>8 \mathrm{mJy}\right)$ with the SMA interferometer and found that the probability of a source being resolved into two or more sources of comparable flux-density is $15 \%$. Simulations of Herschel $500 \mu \mathrm{m}$ surveys have been carried out by Nguyen et al. (2010), Roseboom et al. (2010), Wang et al. (2014b), Valiante et al. (2016) and Bethermin et al. (2017). The Valiante et al. study finds that with a 5- $\sigma$ threshold completeness is $97 \%$ and flux-boost is $2 \%$. The Bethermin et al. (2017) simulation suggests that even allowing for clustering of sources, selection at 5- $\sigma$ ensures that the average flux-boosting at 250, 350 and $500 \mu \mathrm{m}$ is 13,21 and $34 \%$ respectively. We have tested the effect of deboosting by these quantities on our extreme starburst sample (Sect. 6 below) and find that the resulting infrared luminosities and star-formation rates are reduced by a median value of 0.08 dex, an amount that would be almost exactly compensated by changing the Hubble constant from 72 to 67. A few examples have been found of Herschel sources which are identified as distant clusters (e.g. Clements et al. 2014, 2016; Wang et al. 2016) but these tend to be extended or multiple submillimetre sources. It is worth noting that thresholding at $3.5 \sigma$, as has been rather widespread in submillimetre surveys, entails a probability of blended sources four times higher than thresholding at $5 \sigma$.

In conclusion the problems of observing in a confused region of sky (flux-blending, increased positional error, flux-boosting) can be greatly reduced by thresholding at $5-\sigma$. We discuss the issue of blended sources further in Sect. 6 .

\section{Lensed galaxy diagnostics}

One of the most serious issues for cosmological analysis of a submillimetre-selected sample is the high incidence of lensed objects. Negrello et al. (2010) argued that a high proportion of $500 \mu \mathrm{m}$ sources with $S 500>100 \mathrm{mJy}$ are likely to be lensed. Wardlow et al. (2013) showed that, after exclusion of blazers and local spirals, more than $78 \%$ of such sources are confirmed lensed sources. Negrello et al. (2010) plotted SEDs of confirmed lensed sources and showed that at optical and near infrared wavelengths we see the lensing galaxy while at submillimetre wavelengths we see emission from the lensed galaxy. Rowan-Robinson et al. (2014) modelled SEDs of 300 Herschel sources in the Lockman-SWIRE area and identified 36 candidate lensed galaxies in this way. They showed how lensing candidates can be extracted by a set of colour-colour constraints 


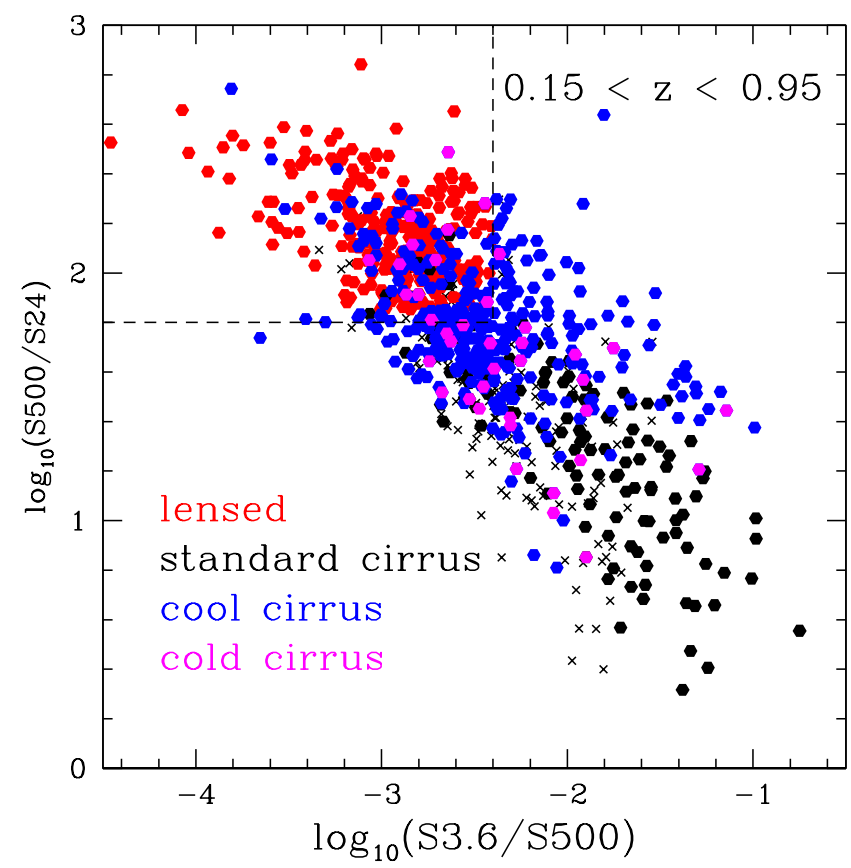

Fig. 1. $S_{24} / S_{500}$ vs. $S_{3.6} / S_{500}$, illustrating the diagnostic ratios used by Rowan-Robinson et al. (2014) to select lensed objects, here plotted for the sources in HerMES (Lockman+XMM+ELAIS S1+CDF-S+ELAIS N1) with $0.15<z<0.95$. Red filled circles: lensed galaxy candidates,large black filled circles: galaxies with standard cirrus components, blue filled circles: galaxies with cool cirrus components, magenta filled circles: galaxies in Lockman with cold cirrus components, small black dots: non-cirrus galaxies.

(including submillimetre colour constraints suggested by Wardlow et al. (2013)).

Figure 1 illustrates the 3.6-24-500 $\mu$ m diagnostic ratios used by Rowan-Robinson et al. (2014) to select lensed objects. It is a plot of $S 500 / S 24$ versus $S 3.6 / S 500$, with candidate lensed objects shown in red, normal cirrus galaxies shown in black, galaxies with cool dust $\left(T_{\text {dust }} \sim 14-19 \mathrm{~K}\right)$ shown in blue and galaxies with cold dust ( $T_{\text {dust }} \sim 9-13 \mathrm{~K}$ ) shown in magenta. The colour selection shown, with others, is remarkably effective at identifying lensed galaxy candidates. In particular the two confirmed lenses in the SWIRE-Lockman area studied by Wardlow et al. (2013) satisfy these colour contsraints. Details of the table of the 275 HerMES-SWIRE (Lock+XMM+ES1+CDFS+EN1) lensed galaxy candidates are given online ${ }^{2}$. These sources are not used in the subsequent analysis. ALMA or HST imaging would be highly desirable to confirm the reality of these lensed galaxy candidates.

For IRAS FSS (RIFSCz) sources we can not use this colourcolour diagnostic. Instead the infrared luminosity, or inferred star-formation rate, is a good indicator of lensing. There do not seem to be any cases where the true, unlensed star-formation rate is $>10^{4.5} M_{\odot} \mathrm{yr}^{-1}$ (see Fig. 2R). Table 5 lists $22 \mathrm{RIFSCz}$ objects with star-formation rate, calculated by the automated template-fitting code, $>10^{4.5} M_{\odot} \mathrm{yr}^{-1}$. Four are known lenses. One $(\mathrm{F} 14218+3845)$ has been imaged with HST and shows no evidence of lensing (Farrah et al. 2002): Rowan-Robinson \& Wang (2010) point out that there is a discrepancy between the ISO $90 \mu \mathrm{m}$ flux and the IRAS 60 and $100 \mu \mathrm{m}$ fluxes and if the former is adopted a much lower SFR $\left(4400 M_{\odot} \mathrm{yr}^{-1}\right)$ is obtained. Three are blazars, for which the submillimetre emission is

\footnotetext{
$\overline{2}$ http://WWW.mattiavaccari.net/df/mrr/readmespirerev
}

non-thermal, one object is more probably associated with a $z=0.032$ Zwicky galaxy, and three have photometric redshifts greater than four, which their SEDs show are implausible: these seven have been removed from Fig. 2R. We are left with ten new candidate lenses, of which five have spectroscopic redshifts. These 22 sources have been removed from the subsequent analysis.

\section{Stellar mass, dust (and gas) mass, star formation rate}

We have derived stellar masses, dust \& gas masses, and star formation rates for both the RIFSCz and HerMES sources by fitting model spectral energy distributions (SEDs) to the catalogue data. Our approach of fitting optical and near infrared SEDs with templates based on stellar synthesis codes (Babbedge et al. 2006; Rowan-Robinson et al. 2008) allows us to estimate stellar masses. The templates are derived using simple stellar populations, each weighted by a different star formation rate and specified extinction (Berta et al. 2004). An empirical correction is applied to allow for the variation of mass-to-light ratio with age (Rowan-Robinson et al. 2008). A Salpeter massfunction is assumed. Similarly, fitting mid infrared, far infrared and submillimetre data with templates based on radiative transfer models (Efstathiou et al. 2000; Efstathiou \& Rowan-Robinson 2003; Rowan-Robinson \& Wang 2010; Rowan-Robinson et al. 2013, 2016), allows us to estimate star formation rates and dust masses.

In the automated fitting of infrared SED templates and calculation of infrared luminosities and other derived quantities, we previously normalised the SEDs at $8 \mu \mathrm{m}$, if the source was detected there, or at $24 \mu \mathrm{m}$ otherwise. In studying the SEDs of galaxies with very high star-formation rates, we have found that normalisation at $8 \mu \mathrm{m}$ for sources at $z=1.5-3.5$ can result in poor estimates of the infrared luminosity, because for many sources with $z>1.5$ the $8 \mu \mathrm{m}$ emission is dominated by starlight. For $z>3.5$ we already required normalisation to be at $24 \mu \mathrm{m}$ (for this reason).

We have therefore switched to normalisation (and luminosity estimation) based on a least-squares fit at $24-500 \mu \mathrm{m}$ for all sources. We have required a $24 \mu \mathrm{m}$ detection in order to associate a Herschel source with a SWIRE photometric redshift catalogue source, so all sources have 24,350 and $500 \mu \mathrm{m}$ detections. This change significantly reduces the number of very high luminosity (and high star-formation rate) galaxies. From detailed SED modelling, we estimate the uncertainty in our corrected luminosities and star-formation rates as $\pm 0.1 \mathrm{dex}$. The star-formation rates are calculated for a $0.1-100 M_{\odot}$ Salpeter IMF. Changing to a Miller-Scalo IMF would increase the star-formation rates by a factor 3.3 , while changing the mass range to $1.6-100 M_{\odot}$, ie forming A, B, O stars only, would reduce them by a factor 3.1 (Rowan-Robinson et al. 1997).

Figure 2L shows our revised plot of star-formation rate (SFR) against redshift for HerMES-SWIRE galaxies, which can be compared with Fig. 2L of Rowan-Robinson et al. (2016). Details of the revised HerMES-SWIRE catalogue are given online $^{3}$. The revised luminosities have some effect on the bright end of the star-formation rate functions. In Fig. 3 we show the star-formation rate functions for $z=0.75-3.25$, derived using the new least-squares normalisation. The tendency of the bright end of the function to be overestimated relative to the model fits

\footnotetext{
3 http://www . mattiavaccari.net/df/mrr/readmespirerev
} 

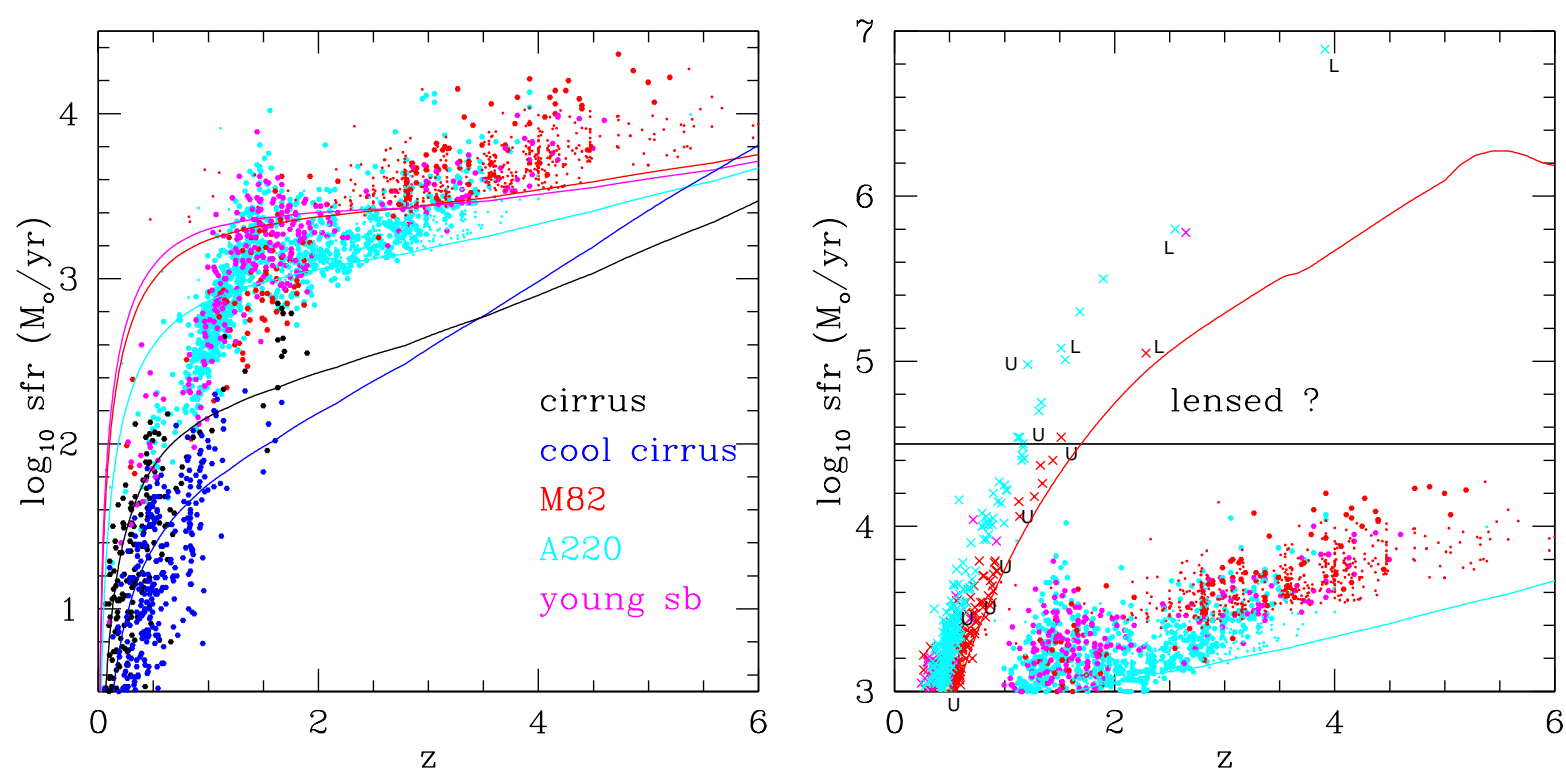

Fig. 2. Left panel: star formation rate versus redshift for HerMES Lockman+XMM+ES1 galaxies, with loci showing $500 \mu \mathrm{m}$ selection limits for each template type. Small dots are unidentified sources. Right panel: star-formation rate versus redshift for extreme starbursts from both HerMES (Lockman+XMM+ES1, filled circles) and from the IRAS RIFSCz catalogue (crosses). Known lenses are indicated by L and cases known to be unlensed indicated by $\mathrm{U}$. The unlensed object apparently above the $30000 M_{\odot} \mathrm{yr}^{-1}$ line (F14218+8345) is discussed in Sect. 4 . Typical 60 and $500 \mu \mathrm{m}$ selection limits are indicated by the red and cyan loci.

Table 5. RIFSCz objects with apparent SFRs $>10^{4.5} M_{\odot} \mathrm{yr}^{-1}$.

\begin{tabular}{|c|c|c|c|c|c|}
\hline IRAS name & $\mathrm{RA}(\mathrm{J} 2000)$ & $\operatorname{Dec}(\mathrm{J} 2000)$ & Redshift & $\begin{array}{l}\log _{10} \mathrm{SFR} \\
\left(M_{\odot} \mathrm{yr}^{-1}\right)\end{array}$ & Notes \\
\hline \multicolumn{6}{|c|}{ Candidate lensed objects } \\
\hline F02416-2833 & 40.953415 & -28.343891 & 1.514000 & 4.54 & \\
\hline F03445-1359 & 56.718334 & -13.844521 & $(1.14)$ & 4.54 & \\
\hline $\mathrm{F} 08105+2554$ & 123.380363 & 25.750853 & 1.512380 & 5.08 & Lensed \\
\hline $\mathrm{F} 08177+4429$ & 125.316353 & 44.333546 & $(2.65)$ & 5.78 & \\
\hline $\mathrm{F} 08279+5255$ & 127.923744 & 52.754921 & 3.912200 & 6.89 & Lensed \\
\hline $\mathrm{F} 10018+3736$ & 151.207672 & 37.362133 & 1.684160 & 5.30 & \\
\hline F10026+4949 & 151.469330 & 49.579998 & 1.120000 & 4.54 & Unlensed \\
\hline F10119+1429 & 153.657822 & 14.251303 & 1.550000 & 5.01 & \\
\hline F10214+4724 & 156.144012 & 47.152695 & 2.285600 & 5.05 & Lensed \\
\hline F10534+3355 & 164.055649 & 33.661686 & $(1.17)$ & 4.50 & \\
\hline $\mathrm{F} 13445+4128$ & 206.656906 & 41.225357 & $(1.33)$ & 4.75 & \\
\hline $\mathrm{F} 13510+3712$ & 208.286133 & 36.964321 & 1.311000 & 4.70 & \\
\hline $\mathrm{F} 14132+1144$ & 213.942673 & 11.495399 & 2.550000 & 5.80 & Lensed \\
\hline $\mathrm{F} 14218+3845$ & 215.981201 & 38.530708 & 1.209510 & 4.98 & Unlensed, see text \\
\hline $\mathrm{F} 23265+2802$ & 352.262146 & 28.312298 & $(1.90)$ & 5.50 & \\
\hline \multicolumn{6}{|c|}{ Wrong ID, wrong redshift or blazars } \\
\hline F02263-0351 & 37.221718 & -3.626988 & 2.055000 & $5.51 ?$ & Blazar \\
\hline $\mathrm{F} 00392+0853$ & 10.453402 & 9.173513 & $(4.62 ?)$ & $7.19 ?$ & Alias at $z=1.4$ \\
\hline $\mathrm{F} 06389+8355$ & 102.896248 & 83.865295 & $(4.50 ?)$ & $6.83 ?$ & Alias at $z=1.4$ \\
\hline $\mathrm{F} 13080+3237$ & 197.619431 & 32.345490 & 0.998010 & $4.52 ?$ & Blazar \\
\hline $\mathrm{F} 15419+2751$ & 236.008347 & 27.697693 & $(2.02)$ & $5.55 ?$ & Zwicky gal $z=0.032$ \\
\hline $\mathrm{F} 16360+2647$ & 249.522308 & 26.694941 & $(4.55 ?)$ & $7.23 ?$ & $z=0.0662 \mathrm{MASS}$ gal at $0.27^{\prime}$ \\
\hline F22231-0512 & 336.446899 & -4.950383 & 1.404000 & $5.25 ?$ & Blazar 3C446 \\
\hline
\end{tabular}

(Fig. 9 of Rowan-Robinson et al. 2016) has disappeared. The new parametric fits give star-formation rate densities that differ from the values of Rowan-Robinson et al. (2016) by $<1 \sigma$. A comparison between our SFRD and those previously reported is also given in Table 7 . The effect on the derived star-formationrate density from $z=0-6$ is negligible. For $z>4.5$ there is no change, but these SFRDs are based almost entirely on sources with no association with SWIRE galaxies and so are very uncertain.

Figure 2R shows the SFR against redshift for HerMESSWIRE and RIFSCz galaxies with SFR $>1000 M_{\odot} \mathrm{yr}^{-1}$. Typical IRAS $60 \mu \mathrm{m}$ and Herschel $500 \mu \mathrm{m}$ detection limits are 

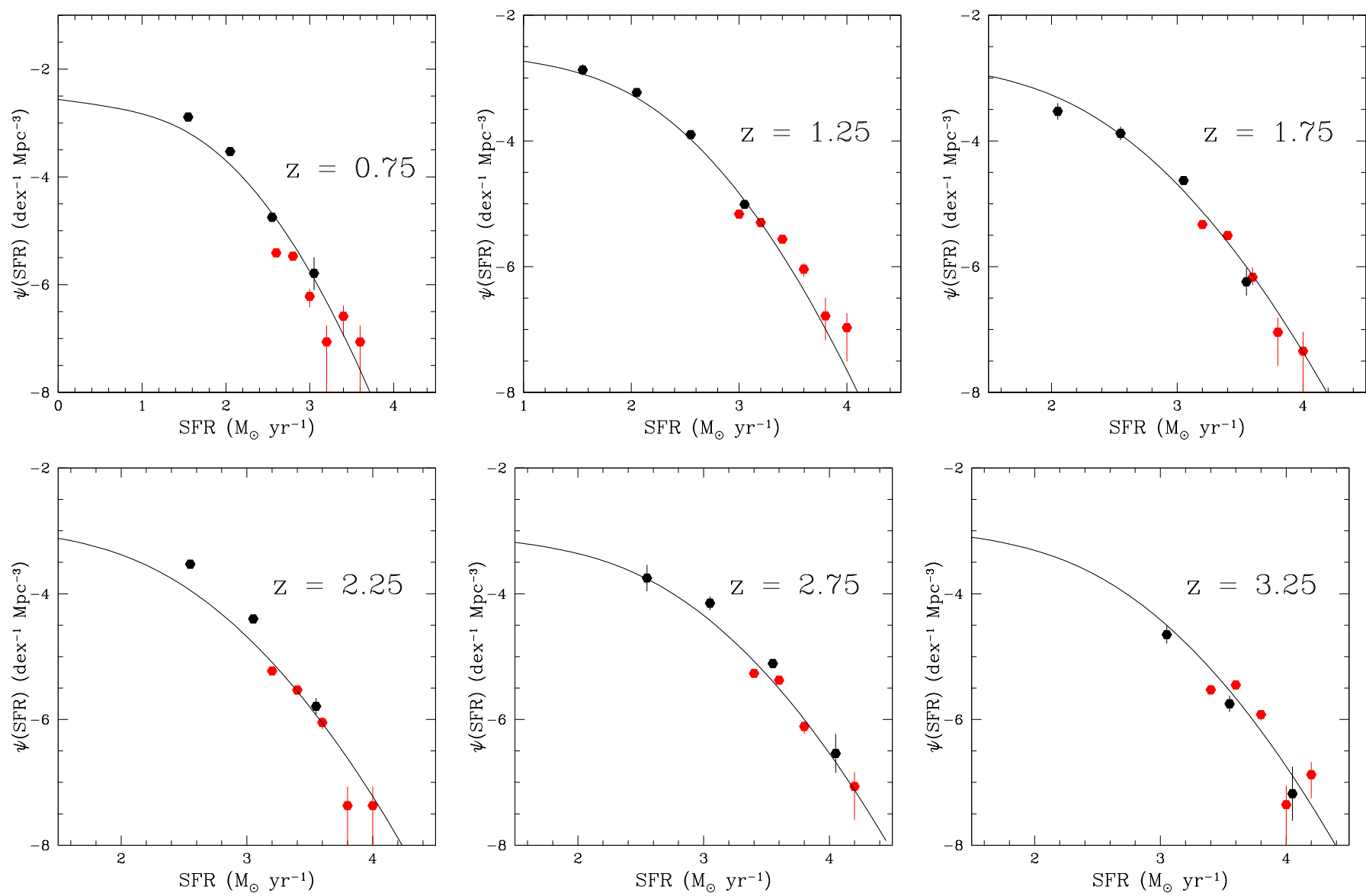

Fig. 3. Revised star-formation rate functions for $z=0.25-3.25$, using a least-squares normalisation at $24-500 \mu \mathrm{m}$. Black dots: data from Gruppioni et al. (2013), red dots: present work.

indicated. The highest star-formation rates significantly exceed the highest rates found by Weedman \& Houck (2008) at $0<\mathrm{z}$ $<2.5$. There appears to be a natural upper limit to the SFR of $30000 M_{\odot} \mathrm{yr}^{-1}$. No HerMES-SWIRE galaxies are found above this value and the IRAS FSS galaxies above this limit are probably gravitational lenses (see previous section and Table 5). This limit could represent an Eddington-type radiation pressure limit on the star-formation rate of the kind postulated by Elmegreen (1983), Scoville et al. (2001), and Murray et al. (2005). Scoville et al. (2001) give a limit for $L / M_{*}$ of $500 L_{\odot} / M_{\odot}$, which would translate to SFR $<10^{4.5} M_{\odot} \mathrm{yr}^{-1}$ for $M_{*}<10^{11.5} M_{\odot}$.

We can use the dust mass as a proxy for gas mass, assuming a representative value for $M_{\text {gas }} / M_{\text {dust }}$. Magdis et al. (2011) have summarised values of $M_{\text {gas }} / M_{\text {dust }}$ as a function of metallicity for local galaxies, and shown that a redshift four galaxy lies on the same relation, with $M_{\text {gas }} \sim 100 M_{\text {dust }}$ (cf. also Chen et al. 2013). We use this ratio to estimate $M_{\text {gas }}$ and then compare this with our stellar mass estimates. Figure 4L illustrates the behaviour of the $\left(M_{\text {gas }}\right) / M_{*}$ ratio as a function of redshift in the HerMES galaxy sample. For HerMES galaxies with redshift greater than one, $M_{\text {gas }}$ is comparable with $M_{\text {stars }}$, so these are very gas-rich galaxies (as noted by Rowan-Robinson et al. 2010). Very high gas fractions have been found in galaxies with redshift greater than one by Daddi et al. (2010), Tacconi et al. (2010, 2013), and Carilli \& Walter (2013). At low redshift, $100 M_{\text {dust }} \sim 0.01-$ $0.1 M_{*}$ so these galaxies have already consumed most of their gas in star-formation.

Figure $4 \mathrm{R}$ shows $M_{*} / \mathrm{SFR}$ as a function of redshift. It is apparent that the time to double the stellar mass at redshift three to five is $\sim 10^{8} \mathrm{yr}$. In some objects the gas-depletion time is as low as $1-3 \times 10^{7} \mathrm{yr}$ (cf. Rowan-Robinson 2000; Carilli \& Walter 2013). The Scoville et al. (2001) Eddington limit quoted above translates to $M_{*} / \mathrm{SFR} \sim 10^{7} \mathrm{yr}$.

The picture that emerges is that the Herschel galaxies at $z>3$ are in the process of making most of the stars in the galaxy. Essentially these are metal factories. However we are not seeing monolithic galaxy formation of the kind postulated by Partridge \& Peebles (1967), even though the star-formation rates and time-scales are similar to those they suggested, because we can see from the optical and near infrared SEDs that there has been an earlier generation of star-formation at least $1 \mathrm{Gyr}$ prior to the star-formation we are witnessing. This is evidenced by the classic 0.4-2 $\mu \mathrm{m}$ SED profile of evolved red giant stars seen in the SEDs of many of these galaxies (cf. Fig. 9 of Bruzual \& Charlot 2003). Between $z=1$ and the present epoch we see a dramatic decline in the gas content and star-formation rate. For $z<0.5$ the gas depletion time-scale is longer than the age of the universe so these are galaxies that must have had a much higher rate of star-formation in the past.

\section{Extreme starbursts}

Here we look in more detail at the galaxies in the HerMESSWIRE survey with implied star-formation rates greater than $5000 M_{\odot} \mathrm{yr}^{-1}$. Previously, detailed studies have been presented of just two objects in this class: Rowan-Robinson \& Wang (2010) show the SED of one unlensed RIFSCz galaxy in this class (IRAS F15307+3252, $z=0.926$ ) with SFR = 

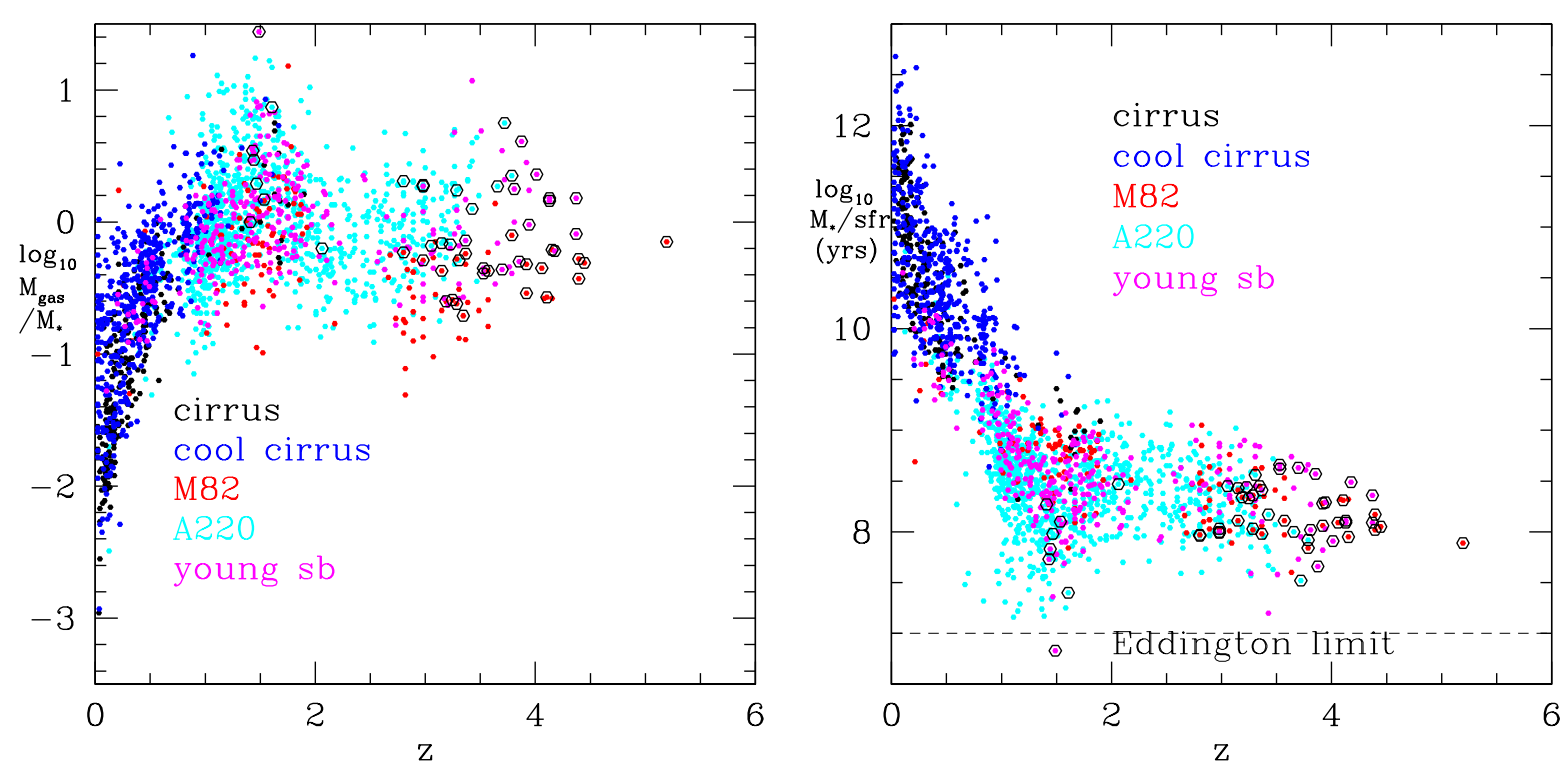

Fig. 4. Left panel: $M_{\text {gas }} / M_{*}$ vs. redshift (where $M_{\text {gas }}=100 M_{\text {dust }}$ ). Circled points are the "extreme" starbursts, those with SFR $>5000 M_{\odot} \mathrm{yr}^{-1}$. Right panel: $M_{*} /$ SFR (or, the time-scale needed to make the observed mass of stars at the present star-formation rate) vs. redshift for HerMES galaxies. The source are labelled by their dominant infrared template type. The candidate extreme starbursts are shown circled.

$8100 M_{\odot} \mathrm{yr}^{-1}$, and Dowell et al report an object (FLS1, $\left.z=4.29\right)$ with $\mathrm{SFR}=9700 M_{\odot} \mathrm{yr}^{-1}$.

Our starting point is the HerMES-SWIRE $\left(\right.$ Lock+XMM+ES1) galaxies with SFR $>5000 M_{\odot} \mathrm{yr}^{-1}$, according to our automated infrared template fitting. There are 70 candidates in all (details given in Tables A.1-A.3), but we have taken a robust approach to the reliability of the redshift estimates, rejecting sources with lower-redshift aliases which give acceptable SED fits, and to the possibility of alternative associations with lower redshift counterparts or blends (see below), resulting in a final list of 38 reliable extreme starbursts. Details of the rejected sources and the reasons for rejection are given in Table A.3. Sources from Table A.3 have been excluded from Figs. 4, 11, 12.

\subsection{Reliability of redshift estimates}

For the 70 candidate objects, we have refined the redshift estimates adopting the approach of Rowan-Robinson et al. (2016), who showed that fitting our starburst templates to the 250$350-500 \mu \mathrm{m}$ data gives an effective estimate of submillimetre redshift, $z_{\text {subm }}$. Combining the $\chi^{2}$ distributions for the photometric and submillimetre redshifts gives a best fit combined redshift $z_{\text {comb }}$. The values of $z_{\text {subm }}$ and $z_{\text {comb }}$ are given in Tables A.1 and A.2. If $z_{\text {comb }}$ is significantly less than $z_{\text {phot }}$ and gives an acceptable SED fit, we have removed the object from the extreme starburst category and the SED is not shown here (17 objects in all). It is possible that in some cases the higher redshift is correct, but we prefer to err on the side of caution. The source 9.17274-43.34398 $\left(z_{\text {phot }}=3.06\right)$ has a spectroscopic redshift of 1.748 , which agrees well with $z_{\text {subm }}$, and so the source has been excluded.

We have also examined the $\chi^{2}$ distribution for the photometric redshift fit to see if any lower redshift aliases are present and the SEDs have also been examined for these aliases. Again we have erred on the side of caution and removed seven objects with lower redshift aliases. 70 and $160 \mu \mathrm{m}$ fluxes have been included in the SED plots only if they have a signal-to-noise ratio of at least four. For three sources (35.73369-5.62305, 7.98209-
43.29812 and $161.89894+58.16401), z_{\text {comb }}$ is significantly less than $z_{\text {phot }}$, but the source remains in the extreme starburst category even with $z=z_{\text {comb }}$, so we have shown the SED with $z_{\text {comb }}$ above the corresponding SED for $z_{\text {phot }}$.

For 36.84426-5.31016 the photometric redshift (3.07) agreed well with the $z_{\text {subm }}$ (3.01) and with $z_{\text {comb }}$ (3.07), but the $\chi^{2}$ for the photometric redshift fit was very poor, and the template fit to the far infrared and submillimetre data was also poor, so we have preferred an alternative association with a SWIRE $z_{\text {phot }}=1.49$ galaxy, which gives a good overall fit to the SED, and so have excluded the object from the extreme starburst category. There are four other objects where detailed modelling of the SED gave solutions differing from the automated fit, which did not confirm them as extreme starbursts.

As a further check on our photometric redshifts we have fitted our extreme starburst sample using the CIGALE code (Burgarella et al. 2005; Noll et al. 2009). For 12 of our objects this yielded a lower preferred redshift. For each of these cases we have examined their overall optical-to-submillimetre SED to see if this alternative redshift provides a plausible fit. One of these we had already omitted due to a lower redshift alias in the photometric redshift $\chi^{2}$ distribution and for one the lower redshift alias still yields a star-formation rate in the extreme category. For two objects the CIGALE redshift offered a plausible alternative fit to the overall SED and these have been excluded.

We should also consider the reliability of the associations of Spitzer 3.6-24 $\mu \mathrm{m}$ sources with optical and near infrared counterparts. Confusion is not an issue at $24 \mu \mathrm{m}$ and the astrometric accuracy of the merged $3.6-24 \mu \mathrm{m}$ sources is $\pm 0.5 \operatorname{arcsec}$ (Shupe et al. 2007; Vaccari 2015). The average number of galaxies to $i=25.5$, the limit of associations considered here, is 0.014 per sq arcsec (Kashikawa et al. 2004), so multiple associations of optical-nir galaxies with Spitzer sources are extremely rare.

Generally the SED fits for the remaining 38 sources are reliable and plausible, though only one is based on an optical spectroscopic redshift. Spectroscopic confirmation of the remaining objects would be highly desirable. Almost all of our 
Table 6. RIFSCz objects with extreme SFRs $\left(>5000 M_{\odot} \mathrm{yr}^{-1}\right)$.

\begin{tabular}{llllll}
\hline \hline IRAS name & RA(J2000) & Dec(J2000) & Redshift & opt type & $\begin{array}{l}\log _{10} \text { SFR } \\
\left(M_{\odot} \mathrm{yr}^{-1}\right)\end{array}$ \\
\hline F00167-1925 & 4.824683 & -19.138355 & $(0.82)$ & Scd & 4.01 \\
F01175-2025 & 19.983685 & -20.172934 & 0.8137 & QSO & 3.92 \\
F02314-0832 & 38.473282 & -8.319294 & 1.1537 & QSO & 4.40 \\
F04099-7514 & 62.201244 & -75.105988 & 0.6940 & E & 3.90 \\
F07523+6348 & 119.230530 & 63.678543 & $(0.77)$ & QSO & 3.79 \\
F08010+1356 & 120.967873 & 13.795245 & $(1.34)$ & Sab & 4.26 \\
F10328+4152 & 158.926239 & 41.615841 & $(0.90)$ & Sab & 3.73 \\
F12431+0848 & 191.435791 & 8.524883 & 0.9380 & Sbc & 4.15 \\
F13073+6057 & 197.320648 & 60.702477 & $(1.01)$ & QSO & 4.22 \\
F13408+4047 & 205.720627 & 40.533772 & 0.9058 & QSO & 3.78 \\
F13489+0524 & 207.858673 & 5.158453 & 0.6202 & E & 3.78 \\
F14165+0642 & 214.784088 & 6.476324 & 1.4381 & QSO & 3.70 \\
F15104+3431 & 228.108719 & 34.336456 & 0.8554 & QSO & 3.95 \\
F15307+3252 & 233.183395 & 32.71295 & 0.9227 & sb & 3.91 \\
F15415+1633 & 235.966370 & 16.406157 & 0.8500 & QSO & 4.01 \\
F16042+6202 & 241.252289 & 61.907372 & $(0.99)$ & Sab & 3.70 \\
F16501+2109 & 253.077240 & 21.078678 & $(1.17)$ & Sab & 3.86 \\
F17135+4153 & 258.781433 & 41.831528 & $(0.90)$ & Sbc & 3.88 \\
F21266+1741 & 322.241943 & 17.914932 & 0.8340 & Sab & 3.74 \\
\hline
\end{tabular}

Table 7. Comparison between our new star-formation rate density, and that previously published in Rowan-Robinson et al. (2016).

\begin{tabular}{|c|c|c|c|c|c|c|c|c|}
\hline Mean Redshift & $0.5-1.0$ & $1.0-1.5$ & $1.5-2.0$ & $2.0-2.5$ & $2.5-3.0$ & $3.0-3.5$ & $3.5-4.0$ & $4.0-4.5$ \\
\hline $\begin{array}{l}\text { Old SFRD }\left(\log _{10}(\phi)\right) \\
\left(M_{\odot} \mathrm{yr}^{-1} \mathrm{Mpc}^{-3}\right)\end{array}$ & $-1.28 \pm 0.21$ & $-0.95 \pm 0.11$ & $-1.06 \pm 0.13$ & $-1.05_{-0.09}^{+0.27}$ & $-0.82_{-0.36}^{+0.18}$ & $-0.99_{-0.46}^{+0.29}$ & $-0.82_{-0.36}^{+0.18}$ & $-0.79_{-0.41}^{+0.14}$ \\
\hline New SFRD & $-1.27 \pm 0.10$ & $-0.93 \pm 0.11$ & $-1.03 \pm 0.18$ & $-0.90 \pm 0.08$ & $-0.99_{-0.07}^{+0.25}$ & $-1.06 \pm 0.18$ & $-0.89_{-0.46}^{+0.21}$ & $-0.85_{-0.58}^{0.09}$ \\
\hline
\end{tabular}

objects with $z_{\text {phot }}$ greater than four have $S 350>S 250$, but it is worth noting that the range of $z_{\text {subm }}$ for our galaxies with $S 250>S 350>S 500$ is $1.16-4.09$, so the latter condition does not imply low redshift.

To summarise the reliability of our redshift estimates for the 38 extreme starbursts: one has a spectroscopic redshift (indicated by four decimal places in Table A.1), a further two have photometric redshift estimates $z \leq 1.5$ determined from at least six photometric bands, so the rms uncertainty in $(1+z)$ is $<4 \%$ and the probability of a catastrophic outlier is $<3 \%$ (Rowan-Robinson et al. 2013). For the 35 remaining objects with $1.5<z_{\text {phot }}<5.2,10$ of which are based on only three or four photometric bands, the photometric redshift estimates are more uncertain, but are in most cases reinforced by the estimates of $z_{\text {subm }}$. Rowan-Robinson et al. (2016) found that the rms uncertainty in $\left(1+z_{\text {comb }}\right) /\left(1+z_{\text {spect }}\right)$ for 28 Herschel galaxies with spectroscopic redshifts is $\sim 21 \%$. From the $\chi^{2}$ distributions for our photometric redshift estimates we have estimated the corresponding redshift uncertainty, and hence estimated the uncertainty in the star-formation estimate. For 35/38 objects the uncertainty is $\leq 0.1 \mathrm{dex}$. This uncertainty could mean that a few of the objects could move out of the extreme starburst category, probably balanced by others whose redshift has been underestimated, but our overall conclusions are unlikely to be significantly affected.

For comparison we have listed the 19 IRAS RIFSCz objects with SFR $>5000 M_{\odot} \mathrm{yr}^{-1}$ in Table 6 (excluding the objects listed in Table 5). We have modelled the SEDs of these objects individually (not shown here). 11 of the 19 objects have spectroscopic redshifts, so for these objects the redshift uncertainty is not a major issue. However the starburst component is usually fitted out to only 60 or $100 \mu \mathrm{m}$ so the star-formation rates are uncertain by a factor of $\sim 2$. It would be valuable to observe these galaxies at submillimetre wavelengths.

\subsection{Source blending, reliability of SWIRE associations}

Because we threshold at $5 \sigma_{\text {tot }}$, where $\sigma_{\text {tot }}$ is the total noise including confusion noise, problems of source blending and confusion will be reduced (Sect. 3). As a check of this, for each of our $500 \mu \mathrm{m}$ sources we looked at any other possible associations with $24 \mu \mathrm{m}$ SWIRE sources within our 20 arcsec search radius. For 18 of our 38 sources there was only one $24 \mu \mathrm{m}$-detected SWIRE photometric redshift catalogue counterpart within our 20 arcsec search radius. For the remainder we have summed the predicted $500 \mu \mathrm{m}$ fluxes (based on template fitting to the SWIRE data) for all $24 \mu \mathrm{m}$-detected counterparts within the search radius and estimated the fraction of the predicted flux provided by our selected association. For $34 / 38$ sources this fraction is $\geq 90 \%$ and for a further 2 sources it is in the range $80-90 \%$. The overwhelming majority of the alternative associations are unlikely to contribute significantly to the observed $500 \mu \mathrm{m}$ flux.

We looked at the SEDs of all the alternative associations to see whether any of these provided plausible SEDs when combined with our submillimetre sources. For five of our 38 sources there was a plausible alternative lower redshift 
Table 8. Radio positional offsets and $q$-values for extreme starbursts.

\begin{tabular}{llllllll}
\hline \hline RA(J2000) & Dec(J2000) & Frequency & Flux-density & $\begin{array}{l}\text { Offset } \\
\operatorname{arcsec}\end{array}$ & Reference & $q$ & $\begin{array}{l}\log _{10} \mathrm{SFR} \\
\left(M_{\odot} \mathrm{yr}^{-1}\right)\end{array}$ \\
\hline 36.10986 & -4.45889 & $1.4 \mathrm{GHz}$ & $0.219 \pm 0.026 \mathrm{mJy}$ & 0.5 & $(1)$ & 2.46 & 4.20 \\
161.75087 & 59.01883 & $324.5 \mathrm{MHz}$ & $687 \pm 72 \mu \mathrm{Jy}$ & 0.7 & $(2)$ & 2.36 & 3.75 \\
& & $1.4 \mathrm{GHz}$ & $278.8 \pm 15.2 \mu \mathrm{Jy}$ & 1.2 & $(3)$ & & \\
& & $1.2 \mathrm{~mm}$ & $3.5 \pm 0.6 \mathrm{mJy}$ & 1.5 & $(4)$ & & \\
161.98271 & 58.07477 & $1.4 \mathrm{GHz}$ & $0.125 \mathrm{mJy}$ & 1.1 & $(6)$ & 2.12 & 3.76 \\
162.33324 & 58.10657 & $1.4 \mathrm{GHz}$ & $1.36 \mathrm{mJy}$ & 1.1 & $(6)$ & 2.45 & 3.73 \\
162.46065 & 58.11701 & $1.4 \mathrm{GHz}$ & $0.064 \mathrm{mJy}$ & 1.7 & $(6)$ & 2.50 & 3.88 \\
162.91730 & 58.80596 & $324.5 \mathrm{MHz}$ & $1013 \pm 96 \mu \mathrm{Jy}$ & 1.6 & $(2)$ & 2.47 & 3.75 \\
& & $1.4 \mathrm{GHz}$ & $0.504 \mathrm{mJy}$ & 1.0 & $(6)$ & & \\
164.64154 & 58.09799 & $1.4 \mathrm{GHz}$ & $0.182 \mathrm{mJy}$ & 0.7 & $(6)$ & 2.37 & 3.72 \\
\hline
\end{tabular}

References. (1): Bondi et al. (2003), (2): Owen et al. (2009), (3): Owen \& Morrison (2008), (4): Lindner et al. (2011), (5): Geach et al. (2017), Hill et al. (2018) (6): Prandoni (2017, priv. comm.).

association and these could be possible cases of misidentification or blending. These have been shown in Tables A.1 and A. 2 in brackets. One of these sources $(164.64154+58.09799)$ has a radio association (see below and Table 7) which strongly supports the higher redshift association (the radio position is 0.7 arcsec from high redshift position, 15 arcsec from lower redshift position). For two other sources $(164.28366+58.43524$ and $164.52054+58.30782)$ the separation of the lower-z $24 \mu \mathrm{m}$ association from the Herschel position is much less than for the higher-z association (1.9 and 0.6 arcsec compared with 16.0 and 15.3 arcsec, respectively), so the lower-z association may be correct. For $160.50839+58.67179$ the Herschel position is 4.1 arcsec from our $z_{\text {phot }}=3.81$ association, 6.9 arcsec from an alternative $z_{\text {phot }}=1.08$ association, while for 35.28232-4.1400 the Herschel position is 2.4 arcsec from our $z_{\text {phot }}=3.28$ association, 5.3 arcsec from an alternative $z_{\text {phot }}=2.55$ association, so either association is plausible. We have shown the SEDs of these five alternative associations in Figs. 6-9.

One option would be to split the $500 \mu \mathrm{m}$ (and associated 350 and $250 \mu \mathrm{m}$ fluxes) equally between the two possible associations. If this is done 2 of the 5 objects move out of the extreme starburst category defined here. Thus source blending or misassociation is a relatively small problem in this sample.

Confirmation of the correctness of our SWIRE associations with the $250-350-500 \mu \mathrm{m}$ sources can be found through radio maps of some of these sources. Table 8 lists seven of the 38 extreme starbursts for which we have radio data. The positional offsets of the radio sources from the SWIRE $(3.6-24 \mu \mathrm{m})$ positions are all $<1-2$ arcsec. We can also calculate the q-values for these sources, where $q=\log _{10}\left(L_{\mathrm{FIR}} / L(1.4 \mathrm{GHz})\right)$. These lie in the range $2.0-2.6$, with a mean of 2.33, in good agreement with the values found for lower redshift Herschel galaxies by Ivison et al. (2010) and with the mean value 2.34 found for IRAS galaxies (Yun et al. 2001). We have also shown positional offsets for $1.2 \mathrm{~mm}$ MAMBO observations of $161.75087+59.01883$ by Lindner et al. (2011) and for $850 \mu \mathrm{m}$ observations of the same source by Geach et al. (2017). Hill et al. (2018) have observed the same source with the SMA interferometer and confirm that it is a single source. These fluxes are included in the SED of this source plotted in Fig. 5. This is our best-case object, with a spectroscopic redshift (and $z_{\text {subm }}$ closely agreeing with this), radio confirmation of the SWIRE association and the radio estimate of the star-formation rate agreeing well with that from the submillimetre data $(q=2.36)$.

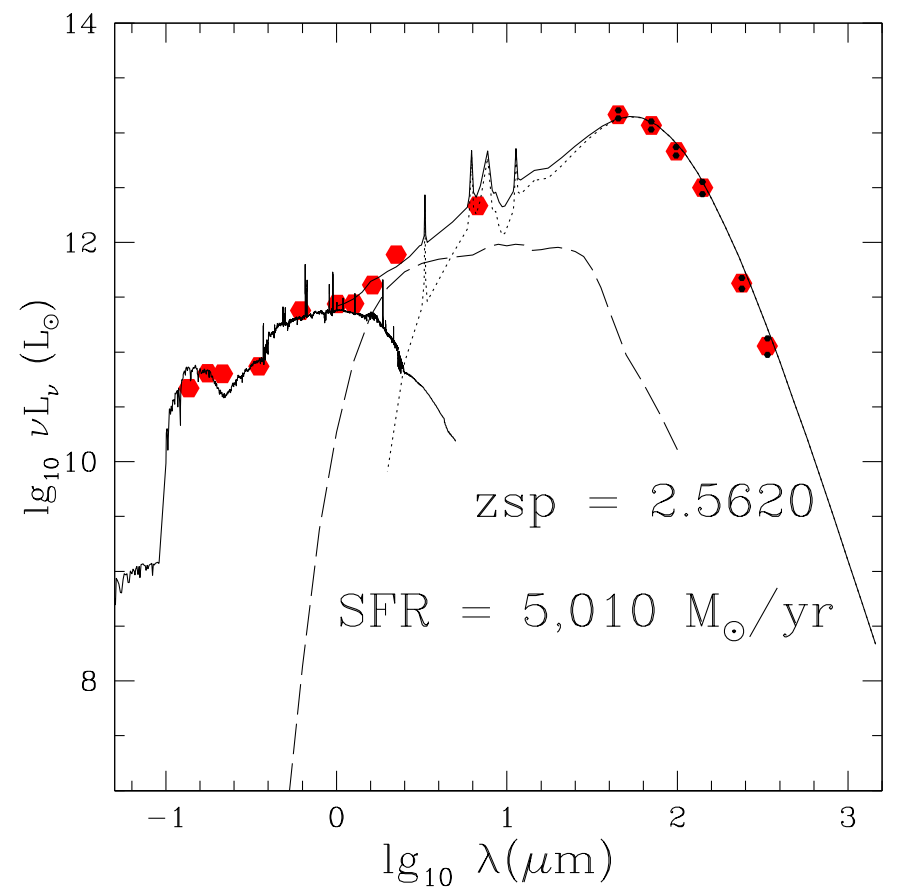

Fig. 5. Rest-frame SED of $161.75087+59.01883$, Herschel-SWIRE $500 \mu \mathrm{m}$ source with spectroscopic redshift and extreme starburst luminosity. SWIRE association is confirmed by radio, $1.2 \mathrm{~mm}$, and $850 \mu \mathrm{m}$ positions (latter two fluxes shown in SED). Errors for submillimetre fluxes indicated by black dots. Dotted loci: M 82 starburst, long-dashed loci: AGN dust torus.

It is worth commenting that the positional uncertainties of our $500 \mu \mathrm{m}$ sample are greatly improved by requiring also 5$\sigma$ detections at $350 \mu \mathrm{m}$. In most cases (34/38) there is a detection at $250 \mu \mathrm{m}$ as well and this is the position used, where available.

While we believe we have presented strong arguments for the reality of these Herschel extreme starbursts, especially those confirmed by radio observations both positionally and in the ratio of far-infrared to radio luminosities, it will be important to confirm the correctness of our SWIRE associations through ALMA and other submillimetre mapping, and through further radio mapping (e.g. by LOFAR, GMRT, MeerKAT and SKA). The correctness of our lensing candidates can be con- 

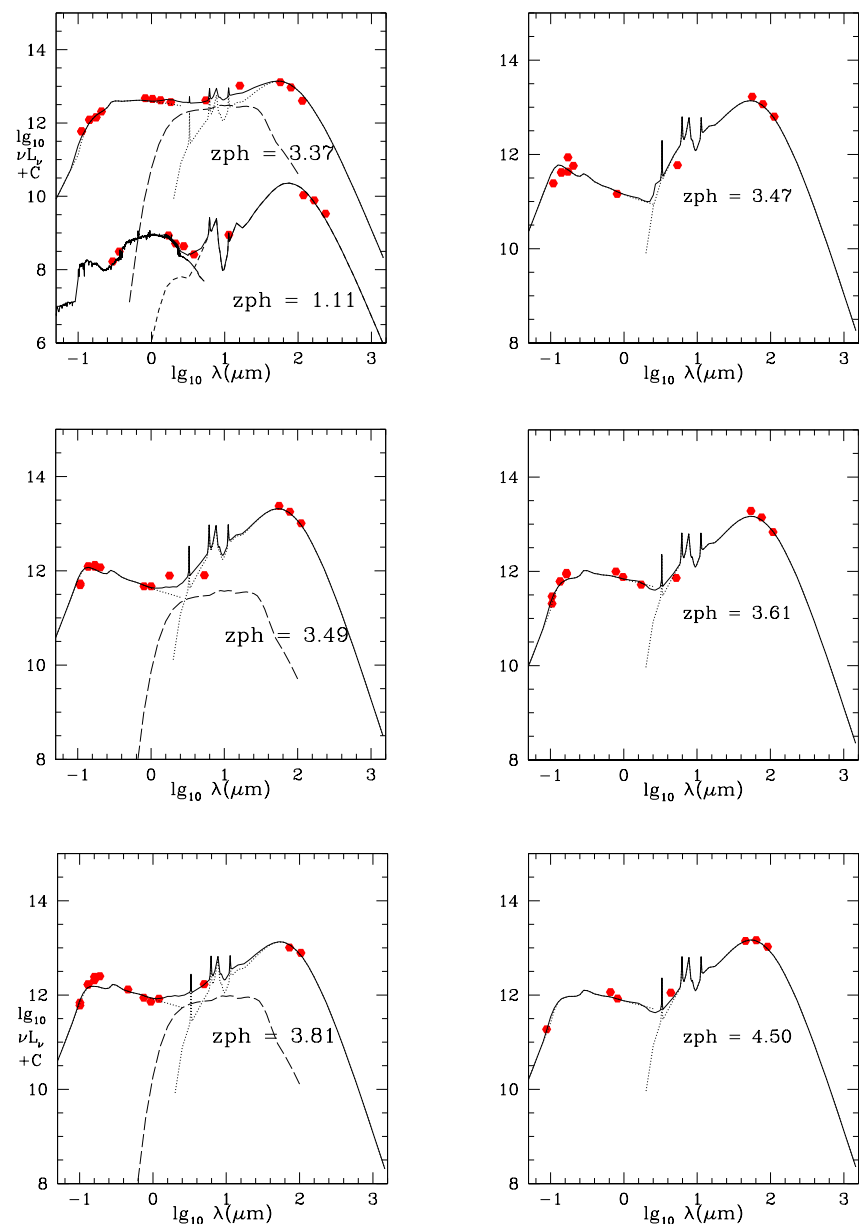

Fig. 6. Rest-frame SEDs of Herschel-SWIRE $500 \mu \mathrm{m}$ sources with extreme starburst luminosities (SFR $>5000 M_{\odot} \mathrm{yr}^{-1}$ ), labelled with the redshift, whose optical through near-infrared SEDs are best-fitted by a QSO template. Alternative SWIRE associations are shown plotted below the extreme starburst solution. Possible redshift aliases $\left(z_{\text {comb }}\right)$ are shown plotted above the preferred $z_{\text {phot }}$ solution. Dotted loci: M 82 starburst, dashed loci: Arp 220 starburst, long-dashed loci: AGN dust torus. The constant $C=0$ except in cases where SEDs are shown for both the photometric redshift and for a lower redshift alternative SWIRE association. In these cases $C=-2$ for the lower SED and +1 for the upper one.

firmed by HST and JWST mapping. For the IRAS extreme starbursts (Table 7) confusion and source blending are not an issue.

In the $2.9 \mathrm{deg}^{2}$ of the SWIRE-CDFS area (not used in this study), we find 8 extreme starbursts, consistent with the surfacedensity of 1.9 per sq deg found in Lockman+XMM+ES1. Unfortunately none of these lie in the $0.25 \mathrm{sq} \mathrm{deg}$ area surveyed at $870 \mu \mathrm{m}$ with LABOCA by Weiss et al. (2009), and followed up with ALMA by Hodge et al. (2013).

\subsection{Role of lensing}

Although we believe we have removed all the lensed systems from our sample (Sect. 4) we need to consider whether any of these 38 extreme starbursts could be lensed. From the analyses of Negrello et al. (2010) and Wardlow et al. (2013) it is the brightest $500 \mu \mathrm{m}$ sources that are most likely to be lensed. None of our extreme starbursts have $S 500>80 \mathrm{mJy}$ but six have $60<S 500<80 \mathrm{mJy}$. Because there is reasonable agreement between $z_{\text {phot }}$ and $z_{\text {subm }}$ for these sources (exact agreement for three of the objects), they could only be lensed if the optical and submillimetre emission was also from the lensed galaxy. This would make them distinct from the known submillimetre lenses. Also three of these bright sources are amongst those confirmed by radio surveys (Table 7) and not reported as lensed. The lensing galaxy candidates found by Rowan-Robinson et al. (2014) typically have $i$-magnitudes in the range 19-22, significantly brighter than the optical counterparts of our extreme starburst sample. We have also checked whether known clusters lie close to any of our 38 objects, in case cluster lensing was an issue, but have found none within one arcmin of our objects. Our expectation is that few or none of our 38 objects will turn out to be lensed systems.

\subsection{SEDs of extreme starbursts}

We present SEDs for these extreme starbursts in the following figures. Figure 6 shows SEDs of extreme starbursts in the Herschel-SWIRE fields whose optical and near infrared data is fitted with a QSO template. Pitchford et al. (2016) have studied a sample of 513 Type 1 QSOs detected by Herschel at $250 \mu \mathrm{m}$, some in the HerMES-SWIRE areas, and found star-formation rates ranging up to $5000 M_{\odot} \mathrm{yr}^{-1}$. In Fig. 7 we show SEDs of objects whose optical-nir data is fitted with a galaxy template, but whose mid ir data show the presence of an AGN dust torus. These sources are plausibly Type 2 AGN whose host galaxies exhibit extreme rates of star formation. Of the 38 objects in our sample, 19 have optical through mid-infrared SEDs consistent with Type 1 or Type 2 AGN. In no case however does the luminosity of the AGN exceed that of the starburst.

In contrast, there exist many examples of "pure" extreme starbursts in our sample. Figure 8 shows SEDs of objects whose optical and near infrared data are fitted with galaxy templates and whose mid ir, far ir and submillimetre data are fitted with M 82 or Arp220 starburst templates. Figure 9 shows an especially interesting set of examples of pure extreme starbursts, whose infrared SEDs are best fit with young starburst templates. None of these objects show any evidence for significant AGN activity. Altogether 19/38 objects are pure starbursts.

As a check on our star-formation rate estimates we have also fitted the overall SEDs with the CIGALE code, using our preferred redshifts. We find that the CIGALE SFR estimates are in broad agreement with ours.

The star formation rates in these extreme starbursts all lie in the range 5000-30000 $\mathrm{M}_{\odot} \mathrm{yr}^{-1}$. As noted in the introduction, such high star formation rates are not predicted by any current semi-analytic model for galaxy formation, so these objects pose a serious challenge to theoretical models. Our $38 \mathrm{Herschel}$ SWIRE objects correspond to a surface density of 1.9 extreme starbursts per sq deg. The $500 \mu \mathrm{m}$ sources which are not associated with SWIRE galaxies could add up to a further $\sim 9$ extreme starbursts per sq deg.

In Tables A.1, A.2 we have also shown our stellar mass estimates. They lie in the range $\lg _{10} M_{*}=11.28-12.50$, so these are exceptionally massive galaxies. For $3.5<z<4.5,11.5<$ $\lg _{10} M_{*}<12.5$, there are 16 objects, yielding a space-density of $10^{-7.12} \mathrm{Mpc}^{-3} \mathrm{dex}^{-1}$, which fits nicely on an extrapolation of the mass-function given by Davidzon et al. 2017, their Figs. 8, 11).

\section{Role of AGN}

A surprisingly high proportion of Herschel extreme starbursts have an inferred AGN dust torus component (50\%). The dust 

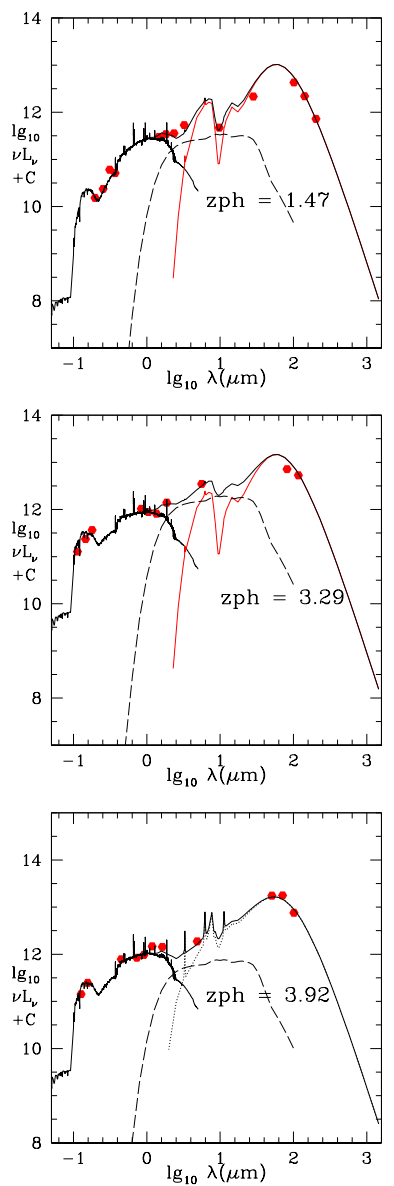
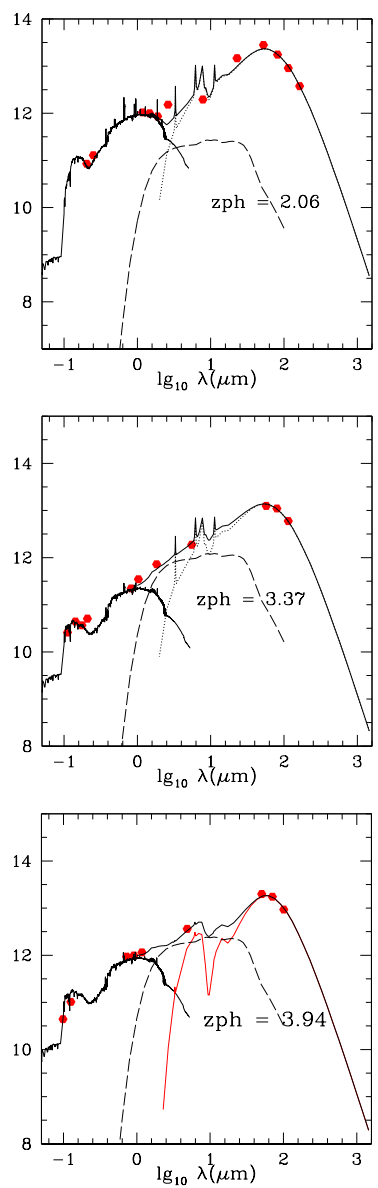
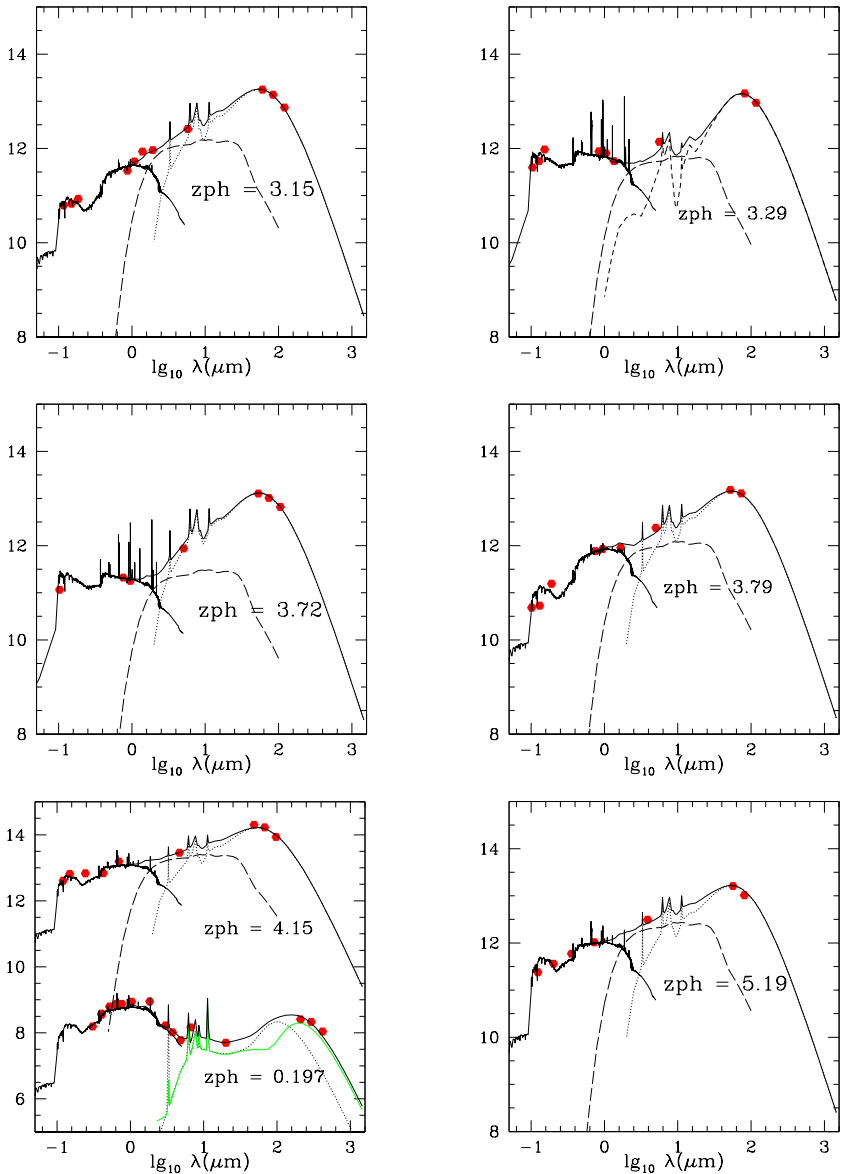

Fig. 7. Rest-frame SEDs of Herschel-SWIRE $500 \mu \mathrm{m}$ sources with extreme starburst luminosities, labelled with the redshift, whose optical through near-infrared SEDs are best-fitted by a galaxy template, but whose mid-infrared SEDs require an AGN dust torus template. Red loci: young starburst template, other details as in Fig. 6.

tori are, however, quite weak and in no case does $L_{\text {tor }}$ exceed $L_{\mathrm{sb}}$, nor does the dust torus contribute significantly to the submillimetre emission. We can use the ratio of luminosity in the dust torus to that in the bolometric uv-optical-nir luminosity of the QSO, $L_{\mathrm{tor}} / L_{\mathrm{bh}}$, as a measure of the covering factor by dust, $f$, which is independent of the geometry of the dust, assuming the thermal uv-optical-nir emission from the accretion disk is radiated isotropically. In the case of a toroidal dust distribution, $f$ would be a measure of the opening angle of the torus. Figure 10 shows $L_{\text {tor }} / L_{\text {opt }}$ versus redshift for SWIRE QSOs, where $L_{\mathrm{opt}}$ is the $0.1-2 \mu \mathrm{m}$ luminosity of the QSO. Assuming the bolometric output of the black hole, $L_{\mathrm{bh}}=2.0 L_{\mathrm{opt}}$ (Rowan-Robinson et al. 2009), the average covering factor, $f$, is $\sim 0.4$ for $z>2$, declining to $\sim 0.16$ at $z=0$. This trend can also be interpreted as a decline in dust torus covering factor with declining optical (and bolometric) luminosity (see Rowan-Robinson et al. 2009 and references quoted therein).

Using this relation, Fig $11 \mathrm{~L}$ shows black-hole mass, $M_{\mathrm{bh}} \beta^{-1}$, versus total stellar mass, $M_{*}$, for Herschel galaxies and for IRAS-FSS galaxies with $z<0.3$, where $M_{\mathrm{bh}}$ is estimated from $L_{\mathrm{bh}}$ assuming that the $\mathrm{AGN}$ is radiating at a fraction $\beta$ of the Eddington luminosity:

$L_{\mathrm{bh}}=\beta L_{\mathrm{Edd}}=4 \pi \beta G M_{\mathrm{bh}} m_{\mathrm{pc}} / \sigma_{\mathrm{T}}=3.2 \times 10^{4} \beta\left(M_{\mathrm{bh}} / M_{\odot}\right)\left(L_{\odot}\right)$

$L_{\mathrm{bh}}$ is estimated as $2.0 L_{\mathrm{opt}}$ for QSOs, and from $L_{\mathrm{tor}} / f$ for galaxies with AGN dust tori. A wide range of values of the Eddington ratio $\beta$ is found in the literature (Babic et al. 2007; Fabian et al. 2008; Steinhardt \& Elvis 2009; Schulze \& Wisotzki
2010; Suh et al. 2015; Pitchford et al. 2016; Harris et al. 2016), with a typical range of 0.01-1 for $z>1$ (Kelly et al. 2010; Lusso et al. 2012). Since QSOs are excluded from Fig. 11L by the requirement for a measurement of stellar mass, these are all Type 2 AGN. The mean value of $\lg _{10} L_{\mathrm{bh}} /\left(\beta M_{*}\right)$ for 500 HerMESSWIRE AGN is -4.11 , with an rms dispersion of 0.56 . There will be a contribution to this rms from the dispersion in values of the covering factor $\mathrm{f}$. The distribution of AGN in Fig. 11L is broadly similar to the equivalent plot by Reines \& Volonteri (2015) for broad-line AGN, though we have a higher proportion of high mass galaxies and we do not have objects corresponding to their elliptical and $S 0$ galaxies.

Figure 11R shows $M_{\mathrm{bh}} \beta^{-1} / M_{*}$ versus redshift for the same galaxies. If we take $\beta \sim 0.1$ as a characteristic value, then $M_{\mathrm{bh}} / M_{*} \sim 0.001$ at all redshifts, with a range of $\sim \pm 1$ dex. This is reminiscent of the Magorrian et al. (1998) relation between black-hole mass and bulge mass (see also review by Kormendy $\&$ Ho 2013). This ratio is set by the very high star-formation (and black-hole build-up) at redshift two to five. The Milky Way, with $M_{*}=6 \times 10^{10} M_{\odot}$ and $M_{\mathrm{bh}}=4 \times 10^{6} M_{\odot}$ lies on the lower end of this range.

Figure 12 shows $M_{\text {acc }} /$ SFR versus redshift for Herschel galaxies and for (non-Herschel) SWIRE galaxies (smaller symbols), where the black-hole accretion rate $M_{\text {acc }}$ is calculated assuming conversion efficiency of accreting mass to radiation is $\epsilon=0.1$ :

$L_{\mathrm{bh}}=\epsilon M_{\mathrm{acc}} c^{2}$ 

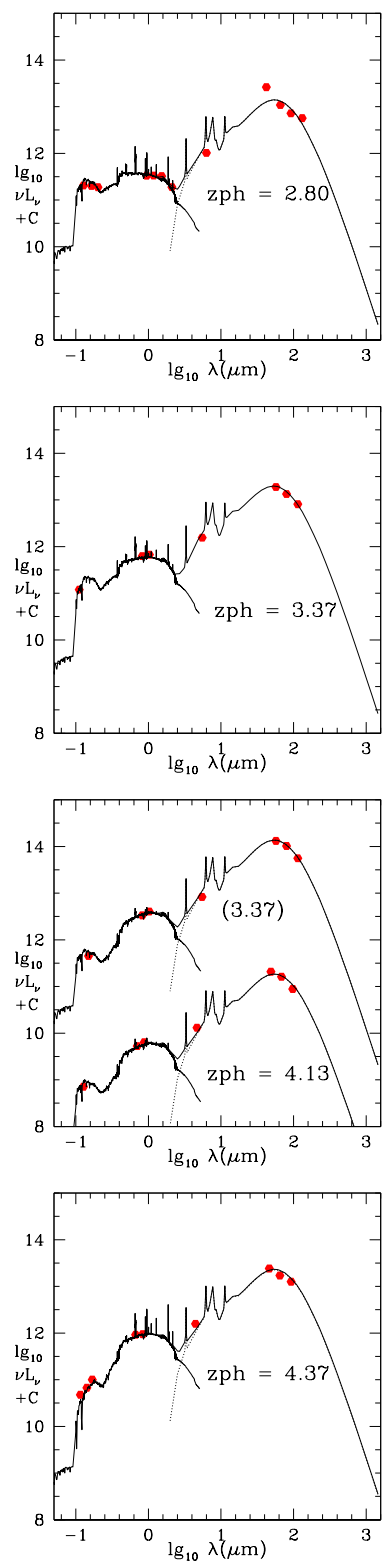
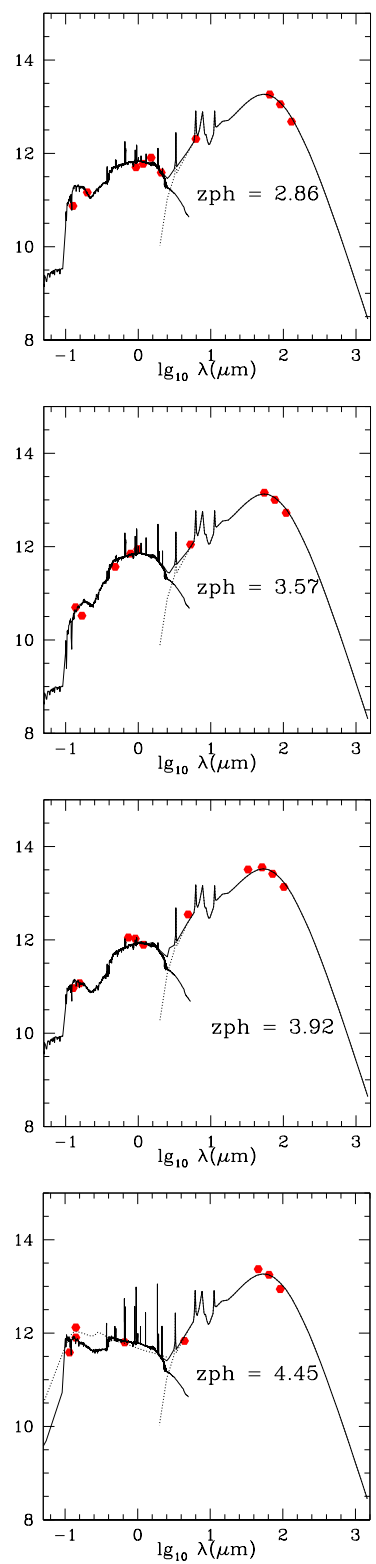
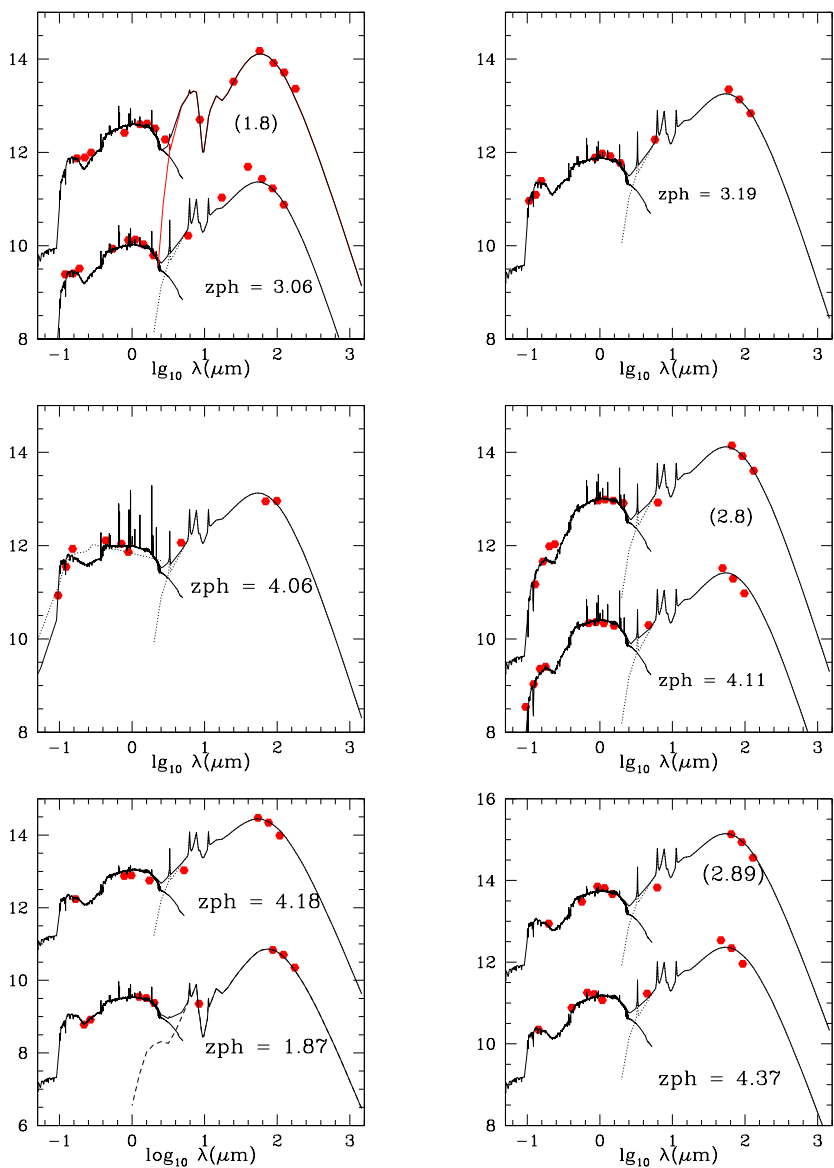

Fig. 8. Rest-frame SEDs of Herschel-SWIRE $500 \mu \mathrm{m}$ sources with extreme starburst luminosities, labelled by redshift, whose optical through nearinfrared SEDs are best-fitted by a galaxy template, and whose mid- through far-infrared SEDs are fitted with M 82 or Arp220 starburst templates. Other details are the same as in Fig. 6.

We note that the combination of Eqs. (6) and (7) gives the Salpeter time-scale for black hole growth $t_{\mathrm{S}}=4.10^{8} \epsilon \beta^{-1} \mathrm{yr}$ (Salpeter 1964). QSOs have been indicated in Fig. 11 by open blue triangles.

Figure 12 shows that $M_{\text {acc }} / \mathrm{SFR}$ is $\sim 10^{-4}$ at $z=2-5$, but that this ratio has increased by a factor of 30 by $z<0.5$. The starformation rates in $z<0.5$ galaxies are 1000 times lower than those seen in the extreme starbursts, but the black hole accretion rates are only 30 times lower. This is consistent with source count models that find shallower evolution for AGN compared to that for starbursts (e.g. Rowan-Robinson 2009). A recent apparent exception to this has been presented by Barnett (2015), who quote a much higher value of $M_{\text {acc }} / \mathrm{SFR} \sim 0.2$ for a redshift 7.1 QSO, based on a SFR derived from the CII $158 \mu \mathrm{m}$ line. However they also quote a bolometric luminosity of $6.7 \times 10^{13} L_{\odot}$, which could yield a SFR of $\sim 13000 M_{\odot} \mathrm{yr}^{-1}$, about 100 times their estimate from CII. This would move $M_{\text {acc }} /$ SFR into the range seen in Fig. 12.
Figure 12 shows that there is an intimate and evolving connection between black hole accretion and star formation. A plausible interpretation of this result is as follows. In these high redshift, high luminosity submillimetre galaxies we are seeing major mergers (Chakrabarti et al. 2008; Hopkins et al. 2010; Hayward et al. 2011; Ivison et al. 2012; Aguirre et al. 2013; Wiklind et al. 2014; Chen et al. 2015), in which the star formation is taking place close to $(<1 \mathrm{kpc})$ the galactic nucleus, so it is not surprising that there is a strong connection between star-formation and black-hole growth. However at recent epochs $(z<1)$ star-formation is mainly fed by accretion from the cosmic web, by minor mergers and interactions, and by spiral density waves, so is taking place further from the galactic nucleus. This uncouples the direct connection between star-formation and black-hole growth. The gas feeding the black hole is fed to the galactic nucleus more gradually and may include gas fed by mass-loss from stars. It is however still surprising that it is so much easier to feed a black hole at the present epoch than it is 

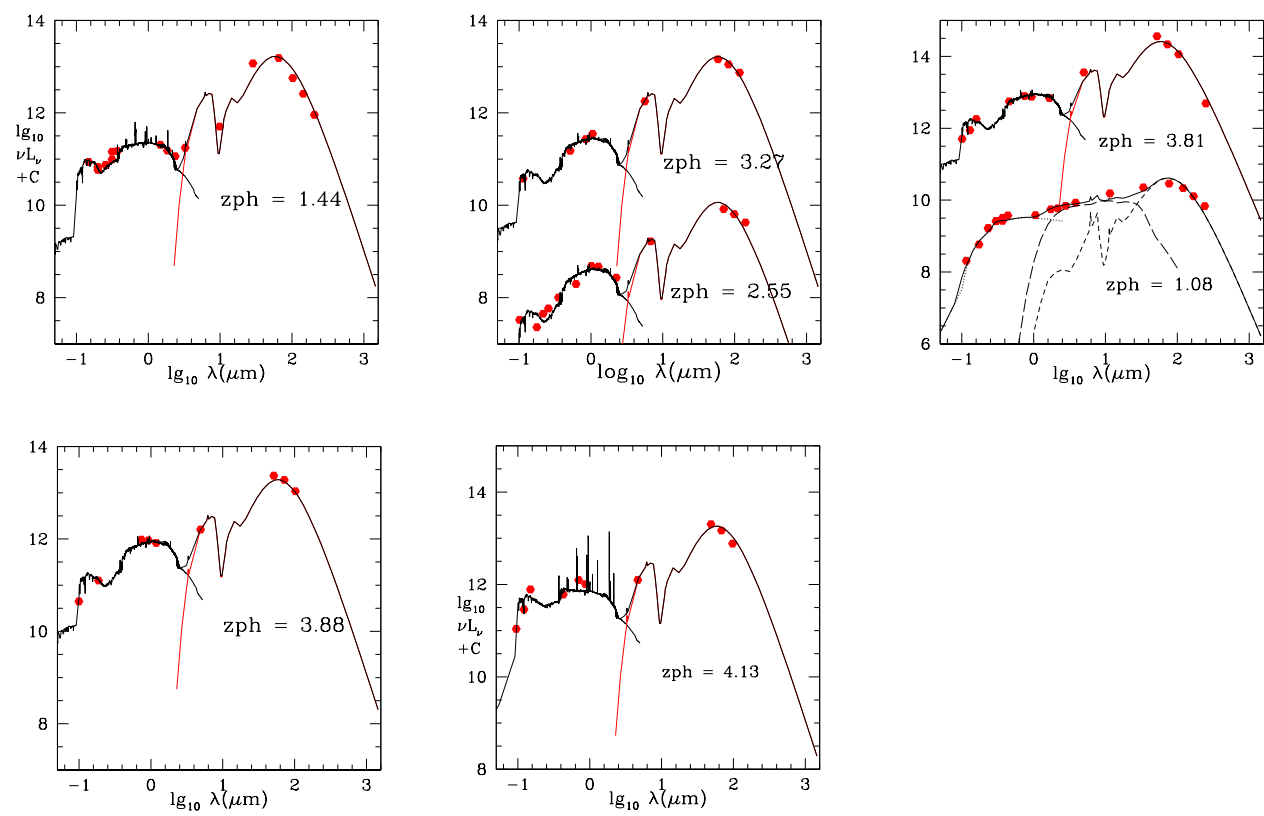

to form stars. Another possible interpretation of Fig. 12 is that the emission from the AGN provides a limit to star-formation, forcing SFR $<10^{5} M_{\text {acc }}$.

It is possible that the high proportion of AGN amongst these extreme starbursts is pointing to the influence of AGN jet-induced star formation in these extreme objects (Klamer et al. 2004; Clements et al. 2009). While we currently have no information on the prevalence of jets in the sample discussed here, there are individual extreme starbursts such as J160705.16+533558.5 (Clements et al. 2009, and in prep.) and 4C41.17 (De Breuck et al. 2005; Steinbring 2014) where there are strong indications that jets are triggering star formation. Furthermore, Klamer et al. (2004) presents a sample of $12 z>3$ star forming AGN where star formation appears to be triggered by relativistic jets. More information on the AGN and gas distribution in the sources in the current paper is clearly needed, but we note that the time-scales for these starbursts and the time-scale for black hole growth are remarkably well matched at $\sim 10^{7} \mathrm{yr}$ (Rigopoulou et al. 2009). However, the greatly enhanced gas supply to the nucleus associated with violent mergers may be a sufficient explanation.

If there is a connection between black hole accretion and star-formation, why do only half of our extreme starbursts harbour AGN ? Firstly the non-detection of an AGN dust torus sets only a modest upper limit on $M_{\mathrm{bh}} \beta^{-1} / M_{*}$ of $\sim 10^{-5}$, which is at the lower end of the observed distribution. It is possible that there is a phase-lag between star-formation and black-hole growth and this is supported by the fact that of the 11 galaxies fitted with a young starburst template in the infrared only two also have an AGN dust torus. To grow a black hole there has to be a black hole present in the first place and perhaps some galaxies have not yet formed a massive nuclear black hole.

Finally, we note an interesting disconnection between X-ray detected AGN, and the Herschel sources. The SWIRE-Lockman area includes the CLASX X-ray survey. Rowan-Robinson et al. (2009) gave a detailed discussion of the associations of CLASX and SWIRE sources. Only two of the 400 CLASX-SWIRE sources are detected by Herschel-SPIRE. This is consistent with the idea that, while AGN are present in the Herschel submillimetre galaxy population, they make a negligible contribution to the submillimetre flux.
Fig. 9. SEDs of Herschel-SWIRE $500 \mu \mathrm{m}$ sources with extreme starburst luminosities, labelled by redshift, whose optical through near-infrared SEDs are best-fitted by a galaxy template, and whose mid- through far-infrared SEDs are fitted with young starburst templates. Red loci: young starburst template. Other details are the same as in Fig. 6.

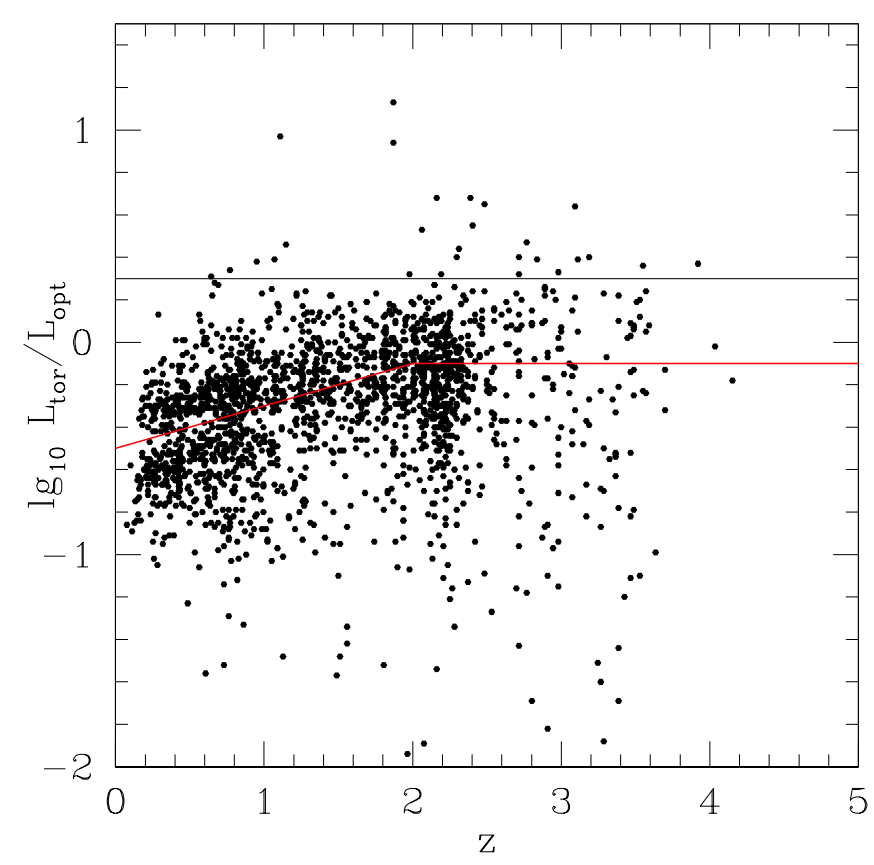

Fig. 10. The behaviour of the torus covering factor $\left(L_{\mathrm{tor}} / L_{\mathrm{opt}}\right)$ as a function of redshift for the HerMES extreme starbursts with QSO-like optical SEDs. The solid black line corresponds to $L_{\mathrm{tor}}=L_{\mathrm{bh}}$ with an assumed optical bolometric correction of 2.0 (Rowan-Robinson et al. 2009). The red line shows a relation that approximately reproduces the trend seen among the plotted population.

\section{Conclusions}

After careful exclusion of lensed galaxies and blazers, we have identified samples of extreme starbursts, with star-formation rates in the range $5000-30000 M_{\odot} \mathrm{yr}^{-1}$, from the IRAS-FSS $60 \mu \mathrm{m}$ galaxy catalogue (RIFSCz) and from the HerschelSWIRE (HerMES) $500 \mu \mathrm{m}$ survey. The correctness of our SWIRE associations is confirmed for 8 objects by radio maps. ALMA submillimetre mapping and deeper radio mapping by LOFAR, GMRT, MeerKAT and SKA will help confirm the reality of the remaining sources. 

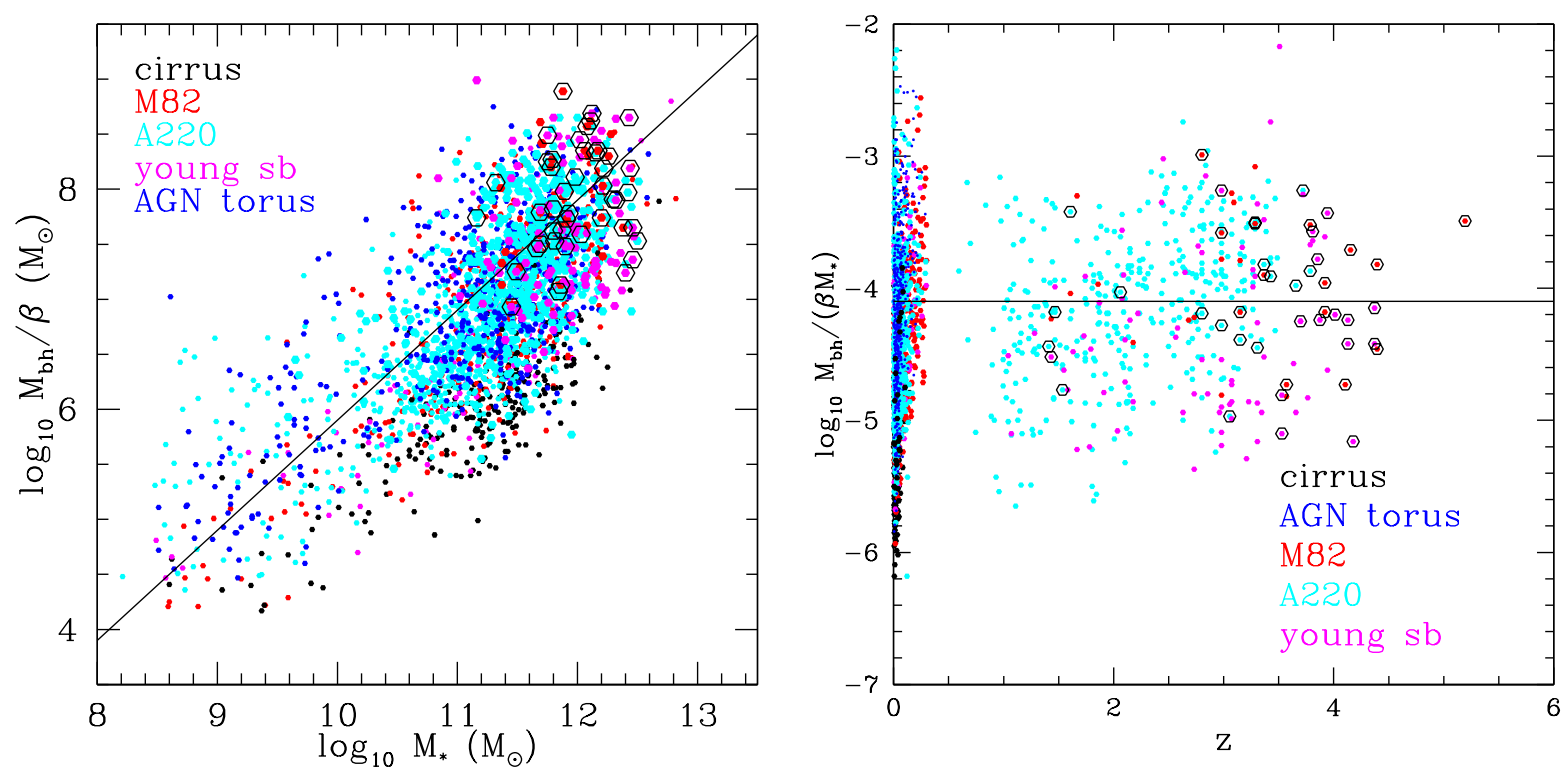

Fig. 11. Left panel: black-hole mass, $M_{\mathrm{bh}} \beta^{-1}\left(M_{\odot}\right)$, versus total stellar mass, $M_{*}\left(M_{\odot}\right)$, for HerMES-SWIRE sources and for RIFSCz sources with $z<0.3$ (smaller points), with AGN dust tori. QSOs are excluded by the requirement that there be a stellar mass estimate, so these are all Type 2 AGN. Circled points are Herschel extreme starbursts. Right panel: $M_{\mathrm{bh}} /\left(\beta M_{*}\right)$ versus $z$ for HerMES-SWIRE sources and for RIFSCz sources with $z<0.3$, with AGN dust tori.

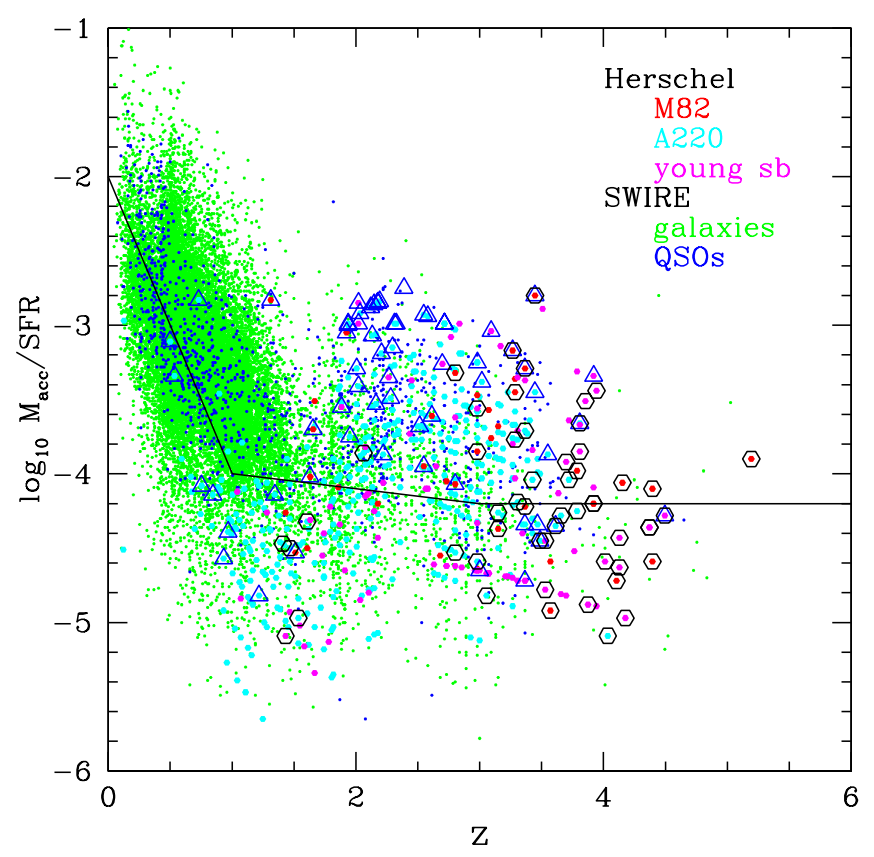

Fig. 12. $\log _{10} M_{\text {acc }} /$ SFR versus $z$ for SWIRE (small green and blue dots) and HerMES sources (larger red cyan and magenta dots). Circled points are extreme starbursts and blue open triangles denote HerMES QSOs.

There do not seem to be any genuine cases with SFR $>30000 M_{\odot} \mathrm{yr}^{-1}$ and this may be essentially an Eddingtontype limit. The SEDs of 38 HerMES extreme starbursts have been modelled in detail. The photometric redshifts are, in almost all cases, supported by redshift estimates from the $250-500 \mu \mathrm{m}$ colours. The proportion of $500 \mu \mathrm{m}$ sources which may be subject to blending or association with the wrong $24 \mu \mathrm{m}$ source is $<12 \%$. Using dust mass as a proxy for gas mass, extreme starbursts are found to be very gas rich systems, which will double their stellar mass in $0.3-3 \times 10^{8} \mathrm{yr}$.

About half of the Herschel extreme starburst systems also contain an AGN, but in no case do these dominate the bolometric output. With assumptions about the Eddington ratio and accretion efficiency, we find a universal relation between black-hole mass and total stellar mass, with $M_{\mathrm{bh}} \sim 0.001 M_{*}$. This is driven by the episode of extreme star-formation and black hole growth at redshift two to five. However while the star formation rate has fallen by a factor of 1000 between redshift five and the present epoch, the black hole accretion rate has fallen by a factor of only 30 , suggesting a decoupling between star formation and the feeding of the nuclear black hole.

Acknowledgements. Herschel is an ESA space observatory with science instruments provided by European-led Principal Investigator consortia and with important participation from NASA. SPIRE has been developed by a consortium of institutes led by Cardiff University (UK) and including Univ. Lethbridge (Canada); NAOC (China); CEA, LAM (France); IFSI, Univ. Padua (Italy); IAC (Spain); Stockholm Observatory (Sweden); Imperial College London, RAL, UCL-MSSL, UKATC, Univ. Sussex (UK); and Caltech, JPL, NHSC, Univ. Colorado (USA). This development has been supported by national funding agencies: CSA (Canada); NAOC (China); CEA, CNES, CNRS (France); ASI (Italy); MCINN (Spain); SNSB (Sweden); STFC, UKSA (UK); and NASA (USA). We thank an anonymous referee for comments that allowed us to substantially improve the paper.

\section{References}

Aaronson, M., \& Olszewski, E. W. 1984, Nature, 309, 414

Aguirre, P., Baker, A. J., Menanteau, F., Lutz, D., \& Tacconi, L. 2013, ApJ, 768, 164

Allen, D. A., Roche, P. F., \& Norris, R. P. 1985, MNRAS, 213, 67

Asboth, V., Coneley, A., Sayers, J., et al. 2016, MNRAS, 462, 1989

Babbedge, T. S. R., Rowan-Robinson, M., \& Vaccari, M. 2006, MNRAS, 370 , 1159

Babic, A., Miller, L., Jarvis, M. J., et al. 2007, A\&A, 474, 755

Barcons, X. 1992, ApJ, 396, 460

Barnett, R. 2015, A\&A, 575, A3

Berta, S., Fritz, J., Franceschini, A., Bressan, A., \& Lonsdale, C. 2004, A\&A, 418, 913

Bethermin, M., Le Floc'h, E., Ilbert, O., et al. 2012, A\&A, 542, A58

Bethermin, M., Wu, H.-Y., Lagache, G., et al. 2017, A\&A, 607, A89

Birnboim, Y., \& Dekel, A. 2003, MNRAS, 345, 349

Blain, A. W., Smail, I., Ivison, R. J., Kneib, J.-P., \& Frayer, D. T. 2002, Phys. Rep., 369, 111

Bondi, M., Ciliegi, P., Zamorani, G., et al. 2003, A\&A, 403, 857 
Bongiorno, R., Maiolino, R., Brusa, M., et al. 2014, MNRAS 443, 2077 Bruzual, G., \& Charlot, S. 2003, MNRAS, 344, 1000

Burgarella, D., Buat, V., \& Iglesias-Paramo, J. 2005, MNRAS, 360, 1413 Carilli, C. L., \& Walter, F. 2013, ARA\&A, 51, 105

Chakrabarti, S., Fenner, Y., Cox, T. J., Hernquist, L., \& Whitney, B. A. 2008, ApJ, 688, 972

Chen, B., Dai, X., Kochanek, C. S., \& Chartas, G. 2013, ArXiv e-prints [arXiv:1306.0008]

Chen, C.-C., Smail, I., Swinbank, A. M., et al. 2015, ApJ, 799, 194

Clements, D. L., Petipas, G., Farrah, D., et al. 2009, ApJ, 698, L188

Clements, D. L., Braglia, F. G., Hyde, A. K., et al. 2014, MNRAS, 439, 1193

Clements, D. L., Braglia, F., Petipas, G., et al. 2016, MNRAS, 461, 1719

Condon, J. J. 1974, ApJ, 188, 279

Daddi, E., Bournaud, F., Walter, F., et al. 2010, ApJ, 713, 686

Dave, R., Finlator, K., Oppenheimer, B. D., et al. 2010, MNRAS, 404, 1355

Davidzon, I., Ilbert, O., Laigle, C., et al. 2017, A\&A, 605, A70

De Breuck, C., Downes, D., Neri, R., et al. 2005, A\&A, 430, L1

Dowell, C. D., Conley, A., Glenn, J., et al. 2014, ApJ, 780, 75

Efstathiou, A., \& Rowan-Robinson, M. 2003, MNRAS, 343, 322

Efstathiou, A., Rowan-Robinson, M., \& Siebenmorgen, R. 2000, MNRAS, 313 734

Eisenhardt, P. R. M., Wu, J., \& Tsai, C. W. 2012, ApJ, 755, 173

Elmegreen, B. G. 1983, MNRAS, 203, 1011

Fabian, A. C., Vasudevan, R. V., \& Gandhi, P. 2008, MNRAS, 385, L43

Farrah, D., Verma, A., Oliver, S., Rowan-Robinson, M., \& McMahon, R. 2002, MNRAS, 329, 605

Franceschini, A. 1982, Ap\&SS, 86, 3

Franceschini, A., Tofflalatti, L., Mazzei, P., Danese, L., \& de Zotti, G. 1991, A\&AS, 89, 285

Geach, J. E., Dunlop, J. S., Halpern, M., et al. 2017, MNRAS, 465, 1789

Gruppioni, C., Pozzi, F., Zamorani, G., \& Vignali, C. 2011, MNRAS, 416, 70

Gruppioni, C., Pozzi, F., Rodighiero, G., et al. 2013, MNRAS, 432, 23

Gruppioni, C., Calura, F., Pozzi, F., et al. 2015, MNRAS, 451, 3419

Hacking, P., \& Houck, J. R. 1987, ApJS, 63, 311

Harris, K., Farrah, D., Schulz, B., et al. 2016, MNRAS, 457, 4179

Hayward, C. C. 2013, MNRAS, 432, L85

Hayward, C. C., Keres, D., Jonsson, P., et al. 2011, ApJ, 743, 159

Henriques, B. M. B., White, S. D. M., Thomas, P. A., et al. 2015, MNRAS, 451, 2663

Hill, R., Chapman, S. C., Scott, D., et al. 2018, MNRAS, 477, 2042

Hodge, J. A., Karim, A., Smail, I., et al. 2013, ApJ, 768, 91

Hogg, D. W. 2001, ApJ, 121, 1207

Hopkins, P. F., Younger, J. D., Hayward, C. C., Narayan, D., \& Hernquist, L. 2010, MNRAS, 402, 1693

Houck, J. R., Scneider, D. P., Danielson, G. E., et al. 1985, ApJ, 290, L5

Ilbert, O., McCracken, H. J., Le Fevre, O., et al. 2013, A\&A, 556, A55

Ivison, R. J., Magnelli, B., Ibar, E., et al. 2010, A\&A, 518, L31

Ivison, R. J., Smail, I., Amblard, A., et al. 2012, MNRAS, 425, 1320

Joseph, R. D., \& Wright, G. S. 1985, MNRAS, 214, 87

Kashikawa, N., Shimasaku, K., Yasadu, N., et al. 2004, PASJ, 56, 1011

Kelly, B. C., Vestergaard, M., Fan, X., et al. 2010, ApJ, 719, 1315

Keres, D., Katz, N., Weinberg, D. H., \& Dave, R. 2005, MNRAS, 363, 2

Klamer, I. J., Ekers, R. D., Sadler, E. M., \& Hunstead, R. W. 2004, ApJ, 612 L97

Koprowski, M. P., Dunlop, J. S., Michalowski, M. J., et al. 2017, MNRAS, 471, 4155

Kormendy, J., \& Ho, L. C. 2013, ARA\&A, 51, 511

Lacey, C. G., Baugh, C. M., Frenk, C. S., et al. 2010, MNRAS, 405, 2

Lacey, C. G., Baugh, C. M., Frenk, C. S., et al. 2016, MNRAS, 462, 3854

Laigle, C., McCracken, H. J., Ilbert, O., et al. 2016, ApJS, 224, 24

Lawrence, A., Walker, D., Rowan-Robinson, M., Leech, K. J., \& Penston, M. V. 1986, MNRAS, 219, 687

Le Floc'h, E., Papovich, C., Dole, H., et al. 2005, ApJ, 632, 169

Lindner, R. R., Baker, A. J., Omont, A., et al. 2011, ApJ, 737, L83

Lonsdale, C. J., Smith, H. E., Rowan-Robinson, M., et al. 2003, PASP, 115, 897
Lusso, E., Comastri, A., Simmons, B. D., et al. 2012, MNRAS, 425, 623 Magdis, G. E., Daddi, E., Elbaz, D., et al. 2011, ApJ, 740, L14

Magorrian, J., Tremaine, S., Richstone, D., et al. 1998, AJ, 115, 2285

Michalowski, M. J., Dunlop, J. S., Koprowski, M. P., et al. 2017, MNRAS, 469, 492

Murray, N., Quataert, E., \& Thompson, T. A. 2005, ApJ, 618, 569 Narayanan, D., Hayward, C. C., Cox, T. J., et al. 2010, MNRAS, 401, 1613

Narayanan, D., Turk, M., Feldmann, R., et al. 2015, Nature, 525, 496

Negrello, M., Hopwood, R., De Zotti, G., et al. 2010, Science, 330, 800

Nguyen, H. T., Schukz, B., Levenson, L., et al. 2010, A\&A, 518, L5

Noll, S., Burgarella, D., Giavannoli, E., et al. 2009, A\&A, 507, 1793

Novak, M., Smolcic, V., Delvecchio, I., et al. 2017, A\&A, 602, A5

Oliver, S. J., Bock, J., Altieri, B., et al. 2012, MNRAS, 424, 1614

Owen, F. N., \& Morrison, G. E. 2008, AJ, 136, 1889

Owen, F. N., Morrison, G. E., Klimek, M. D., \& Greisen, E. W. 2009, AJ, 137, 4846

Partridge, R. B., \& Peebles, P. J. E. 1967, ApJ, 147, 868

Perez-Gonzalez, P. G., Rieke, G. H., Egami, E., et al. 2005, ApJ, 630, 82

Pitchford, L. K., Hatziminaoglou, E., Feltre, A., et al. 2016, MNRAS, 462, 4067

Planck Collaboration XVI. 2014, A\&A, 571, A16

Reines, A. E., \& Volonteri, M. 2015, ApJ, 813, 82

Rigopoulou, D., Mainieri, V., Almaini, O., et al. 2009, MNRAS, 400, 1199

Roseboom, I. G., Oliver, S. J., Kunz, M., et al. 2010, MNRAS, 409, 48

Rowan-Robinson, M. 2000, MNRAS, 316, 885

Rowan-Robinson, M. 2009, MNRAS, 394, 117

Rowan-Robinson, M., \& Clements, D. L. 2015, MNRAS, 453, 2050

Rowan-Robinson, M., \& Wang, L. 2010, MNRAS, 406, 720

Rowan-Robinson, M., \& Wang, L. 2017, Pub. Kor. Astron. Soc., 32, 293

Rowan-Robinson, M., Mann, R. G., Oliver, S. J., et al. 1997, MNRAS, 289, 490

Rowan-Robinson, M., Babbedge, T., Oliver, S., et al. 2008, MNRAS, 386, 697

Rowan-Robinson, M., Valtchanov, I., \& Nandra, K. 2009, MNRAS, 397, 1326

Rowan-Robinson, M., Roseboom, I. G., Vaccari, M., et al. 2010, MNRAS, 409, 2

Rowan-Robinson, M., Gonzalez-Solares, E., Vaccari, M., \& Marchetti, L. 2013, MNRAS, 428, 1958

Rowan-Robinson, M., Wang, L., Wardlow, J., et al. 2014, MNRAS, 445, 3848

Rowan-Robinson, M., Oliver, S., \& Wamg, L. 2016, MNRAS, 461, 1100

Salpeter, E. E. 1964, ApJ, 140, 796

Scheuer, P. A. G. 1957, Proc. Camb. Philos. Soc., 53, 764

Schulz, B., Marton, G., Valtchanov, I., et al. 2017, SPIRE Point Source Catalog Explanatory Supplement (Caltech: IPAC)

Schulze, A., \& Wisotzki, L. 2010, A\&A, 516, A87

Scott, S. E., Fox, M. J., Dunlop, J. S., et al. 2002, MNRAS, 331, 817

Scoville, N. Z., Polletta, M., Ewald, S., et al. 2001, AJ, 122, 3017

Scoville, N., Aussel, H., Benson, A., et al. 2007, ApJS, 172, 1

Shupe, D. L., Alexov, A., Evans, T., et al. 2007, ASPC, 376, 457

Soifer, B. T., Helou, G., Lonsdale, C. J., et al. 1984, ApJ, 283, L1

Steinbring, E. 2014, AJ, 148, 10

Steinhardt, C. L., \& Elvis, M. 2009, MNRAS, 402, 2637

Suh, H., Hasinger, G., Steinhardt, C., Silverman, J. D., \& Schramm, M. 2015, ApJ, 815, 129

Tacconi, L., Genzel, R., Neri, R., et al. 2010, Nature, 463, 781

Tacconi, L., Neri, R., Genzel, R., et al. 2013, ApJ, 768, 74

Vaccari, M. 2015, Proceedings of "The many facets of extragalactic radio surveys: towards new scientific challenges", 27

Valiante, E., Smith, M. W. L., \& Eales, S. 2016, MNRAS, 462, 3146

Wang, L., Rowan-Robinson, M., Norberg, P., Heinis, S., \& Han, J. 2014a, MNRAS, 442, 2739

Wang, L., Viero, M., Clarke, C., et al. 2014b, MNRAS, 444, 2870

Wang, T., Elbaz, D., Daddi, E., et al. 2016, ApJ, 828, 56

Wardlow, J. J., Cooray, A., De Bernadis, F., et al. 2013, ApJ, 762, 59

Weedman, D. W., \& Houck, J. R. 2008, ApJ, 686, 127

Weiss, A., Kovacs, A., Coppin, K., et al. 2009, ApJ, 707, 1201

Wiklind, T., Conselice, T. J., Dahlen, T., et al. 2014, ApJ, 785, 111

Yun, M. S., Reddy, N. A., \& Condon, J. J. 2001, ApJ, 554, 803

Zinn, P.-C., Middelberg, E., Norris, R. P., et al. 2012, A\&A, 544, A38 


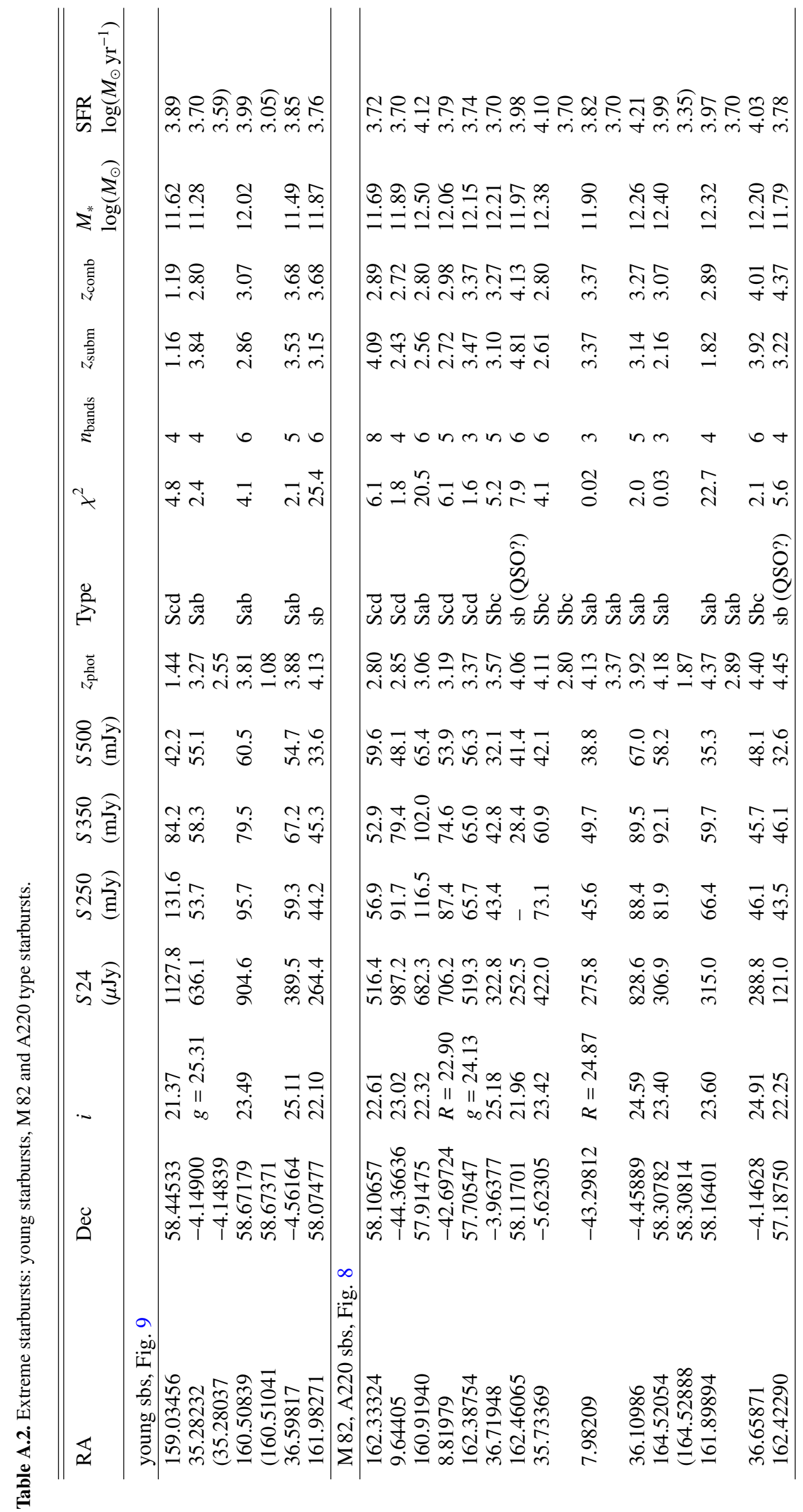




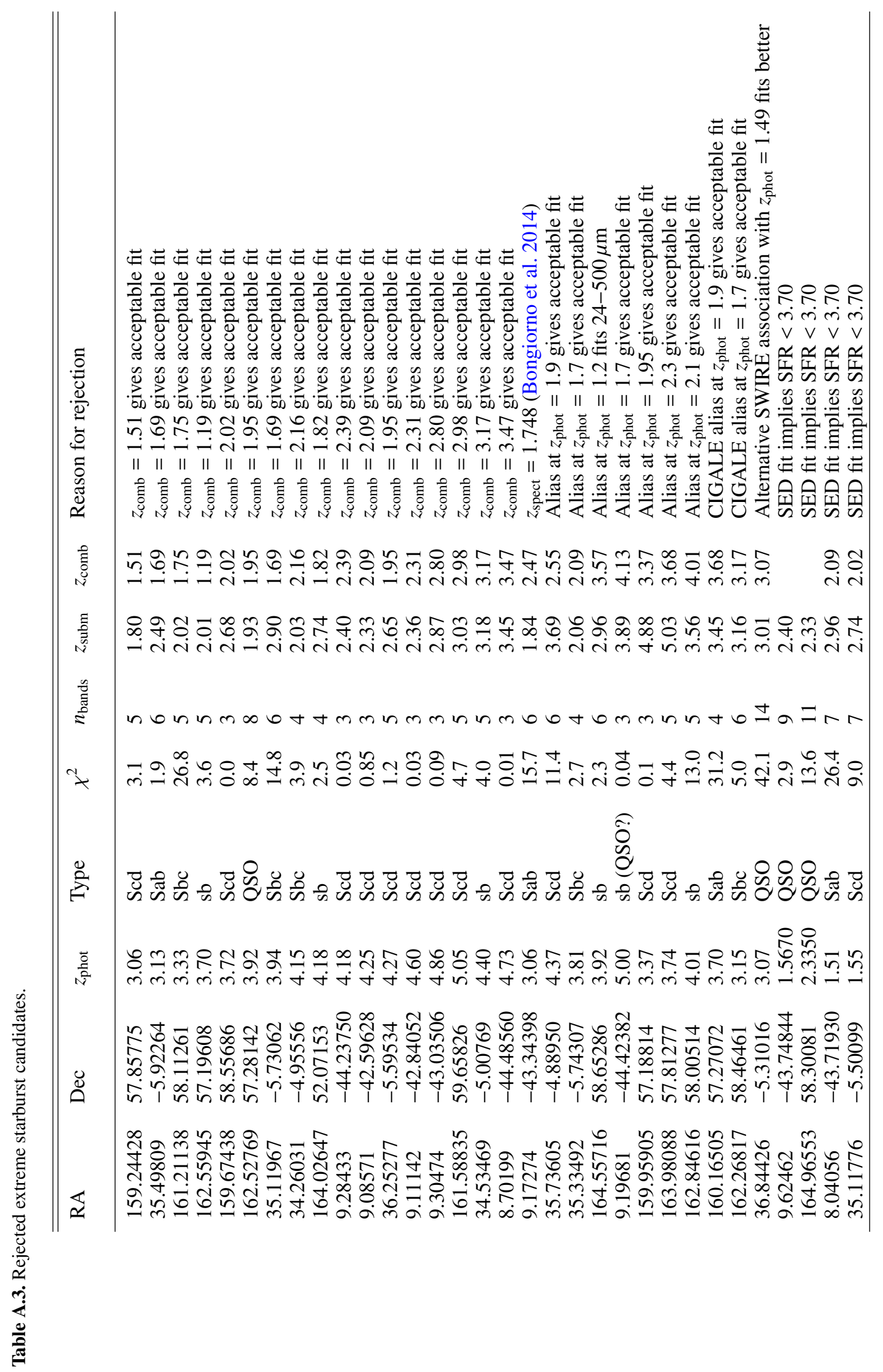

